\title{
THE LUMINOUS INFRARED HOST GALAXY OF SHORT-DURATION GRB 100206A
}

\author{
D. A. Perley ${ }^{1,5}$, M. Modjaz ${ }^{2}$, A. N. Morgan ${ }^{3}$, S. B. Cenko ${ }^{3}$, J. S. Bloom ${ }^{3}$, N. R. Butler ${ }^{4}$, \\ A. V. FilipPENKo ${ }^{3}$, AND A. A. Miller ${ }^{3}$ \\ ${ }^{1}$ Department of Astronomy, California Institute of Technology, MC 249-17, 1200 East California Blvd., \\ Pasadena, CA 91125, USA; dperley@astro.caltech.edu \\ ${ }^{2}$ Center for Cosmology and Particle Physics, New York University, 4 Washington Place, New York, NY 10003, USA \\ ${ }^{3}$ Department of Astronomy, University of California, Berkeley, CA 94720-3411, USA \\ ${ }^{4}$ School of Earth and Space Exploration, Arizona State University, Tempe, AZ 85287, USA \\ Received 2011 December 19; accepted 2012 August 28; published 2012 October 8
}

\begin{abstract}
The known host galaxies of short-hard gamma-ray bursts (GRBs) to date are characterized by low to moderate star formation rates (SFRs) and a broad range of stellar masses, in general agreement with models associating the phenomenon with an old progenitor, such as merging neutron stars. In this paper, we positionally associate the recent unambiguously short-hard Swift GRB 100206A with a disk galaxy at redshift $z=0.4068$ that is rapidly forming stars at a rate of $\sim 30 M_{\odot} \mathrm{yr}^{-1}$, almost an order of magnitude higher than any previously identified short-GRB host. The galaxy is very red $(g-K=4.3 \mathrm{AB} \mathrm{mag})$, heavily obscured $\left(A_{V} \approx 2 \mathrm{mag}\right)$, and has the highest metallicity of any GRB host to date $\left(12+\log [\mathrm{O} / \mathrm{H}]_{\mathrm{KD} 02}=9.2\right)$ : it is a classical luminous infrared galaxy (LIRG), with $L_{\mathrm{IR}} \approx 4 \times 10^{11} L_{\odot}$. While these properties could be interpreted to support an association of this GRB with recent star formation, modeling of the broadband spectral energy distribution also indicates that a substantial stellar mass of mostly older stars is also present. The specific SFR is modest (sSFR $\approx 0.5 \mathrm{Gyr}^{-1}$ ), the current SFR is not substantially elevated above its long-term average, and the host morphology shows no sign of recent merger activity. Our observations are therefore equally consistent with an older progenitor. Given the precedent established by previous short-GRB hosts and the significant fraction of the universe's stellar mass in LIRG-like systems at $z \gtrsim 0.3$, an older progenitor represents the most likely origin of this event.
\end{abstract}

Key words: gamma-ray burst: individual (100206A) - infrared: galaxies

Online-only material: color figures

\section{INTRODUCTION}

Classical gamma-ray bursts (GRBs) segregate into two phenomenological classes on the basis of their prompt-emission properties: long-duration, soft-spectrum GRBs and shortduration, hard-spectrum GRBs (Kouveliotou et al. 1993). The two distributions overlap in both duration and in hardness, but a duration of $T_{90}=2 \mathrm{~s}$ is commonly taken as the dividing line between "long" and "short" bursts. ${ }^{6}$ Long bursts constitute the large majority of events detected by all major GRB satellites (about $75 \%$ of BATSE events and over $95 \%$ of Swift events); because these events have brighter and longerlived afterglows (Kann et al. 2011), they have historically been easier to study. Observations stretching back almost 15 years associate long-duration bursts exclusively with actively starforming host galaxies, and specifically with regions that are bright at ultraviolet (UV) wavelengths (Fruchter et al. 2006) at small offset from the center (Bloom et al. 2002) of these galaxies, strongly pointing toward a massive stellar origin. In a number of cases, evidence for an accompanying supernova has provided direct evidence confirming this conclusion (see Woosley \& Bloom 2006 for a review).

The short-GRB sample is much smaller, but its properties are unambiguously different from those of long bursts. At least two short events (GRBs 050509B and 050724; Bloom

\footnotetext{
5 Hubble Fellow.

6 However, the question of classification has recently become much more complex following the discovery of events with extended emission (Norris \& Bonnell 2006) and deep limits on supernovae from $t>2 \mathrm{~s}$ bursts (Fynbo et al. 2006; Gehrels et al. 2006; Gal-Yam et al. 2006). See Bloom et al. (2008) for a discourse on the subject of classification.
}

et al. 2006; Gehrels et al. 2005; Barthelmy et al. 2005; Berger et al. 2005; Prochaska et al. 2006) have been associated with massive, evolved galaxies having essentially negligible current star formation, pointing strongly toward a long-lived progenitor (Bloom \& Prochaska 2006). In addition, the offset distribution for short GRBs relative to their host galaxies extends to much greater distances than for long GRBs-partially as a simple result of the fact that short-GRB hosts tend to be physically more extended (Fong et al. 2010), but short GRBs also generally show minimal correlation with the host blue/UV light or resolved regions of active star formation (Fox et al. 2005; Fong et al. 2010; Rowlinson et al. 2010). The occurrence of an accompanying bright core-collapse supernova has been definitively ruled out for several short GRBs (e.g., Hjorth et al. 2005; Kocevski et al. 2010).

The model of short GRBs as mergers of compact binary stars (Eichler et al. 1989; Paczyński 1991; Narayan et al. 1992) - either two neutron stars (NS-NS) or a neutron star and a black hole (NS-BH) - naturally predicts old progenitors, and the observations discussed above appear to strongly support this model. However, other models permitting older populations do exist (e.g., MacFadyen et al. 2005), and recent observations have painted a picture that appears more complex than it initially seemed after the discovery of the first few short-GRB hosts. A significant fraction of short GRBs with afterglows actually have no clearly identifiable host (Berger 2010), suggesting that they have been flung large distances from their original hosts or occur in optically underluminous, distant galaxies in surprisingly large numbers. Finally, with the exceptions of GRBs 050509B, 050724, and possibly 100117A (Fong et al. 2011), every shortGRB host to date (at this stage, a fairly formidable sample of 
$>20$ objects) has had at least modest star formation in relation to its stellar mass (e.g., Berger 2010), making it difficult to pin down a minimum progenitor age for the large majority of these events. The two "smoking gun" events of 2005 no longer appear to be particularly representative of the entire class.

The relationship between short and long GRBs is at least vaguely analogous to that between Type Ia and Type II supernovae (e.g., Wheeler 1981): Type Ia supernovae are often associated with moderate or old populations (and almost certainly are produced by compact objects, in this case white dwarfs; Nomoto 1982; Bloom et al. 2012; Nugent et al. 2011), while Type II supernovae are associated exclusively with young populations (and result from massive stellar core collapse). However, recent evidence suggests that Type Ia supernovae can actually be produced fairly rapidly in some cases, given their elevated rate in late-type hosts compared to what might be expected from an exclusively old population (Scannapieco \& Bildsten 2005; Mannucci et al. 2005; Sullivan et al. 2006; Li et al. 2011; Maoz et al. 2011) — that is, the delay-time distribution is likely to be quite broad, with short-lived stellar progenitors as well as longlived ones.

It is possible that the short-GRB delay-time distribution may be similarly complex. Confirming this trend-and in particular, determining whether there is any need for a very young component associated with the youngest (and presumably most massive) stars, which could indicate a physically distinct progenitor (e.g., Metzger et al. 2008; Lazzati et al. 2010; Virgili et al. 2011) - is of clear interest for better understanding the origins of the short-GRB population.

Given the low short-GRB event rate $\left(<10\right.$ events $\left.\mathrm{yr}^{-1}\right)$ and uncertain selection biases affecting the population $(\sim 25 \%$ of all Swift short GRBs are not detected by the X-Ray Telescope (XRT) and cannot be localized), teasing such a tendency out of the full population is challenging. Nevertheless, two recent studies have attempted to accomplish this using different techniques. Virgili et al. (2011) analyzed the short-GRB redshift, fluence, and luminosity distributions compared to predictions from a delay-time convolved star formation history of the universe and concluded that a significant fraction of short GRBs may in fact originate from young stars. Leibler \& Berger (2010) used the properties of the host galaxies of a sample of short GRBs to estimate the age of the predominant stellar population, and similarly concluded that both short-delay and long-delay components are necessitated by the data. However, even the "short" component is consistent with a timescale of the order of $200 \mathrm{Myr}$ - much older than the lifetimes of massive stars that produce core-collapse supernovae, long GRBs, and nebular signatures in star-forming galaxies.

The most unambiguous indicator of a very short-delay $(\lesssim 100 \mathrm{Myr})$ component would be the discovery of another "smoking gun" system, but this time with an extremely young stellar age. In particular, a short GRB within a starbursting galaxy whose current star formation rate (SFR) is very large in comparison to its stellar mass (high specific SFR, sSFR) would be a strong indicator that the event came from a star produced in the starburst, events whose characteristic times rarely exceed a few hundred Myr and are often much less (Di Matteo et al. 2008; McQuinn et al. 2009, 2010).

In this paper, we discuss the case of GRB 100206A as the first example of a short-duration burst for which the characteristics of its probable host, at least on the surface, evoke such a system. In Section 2, we present our observations of the burst and its afterglow, showing it to be an unambiguous short-duration, hard-

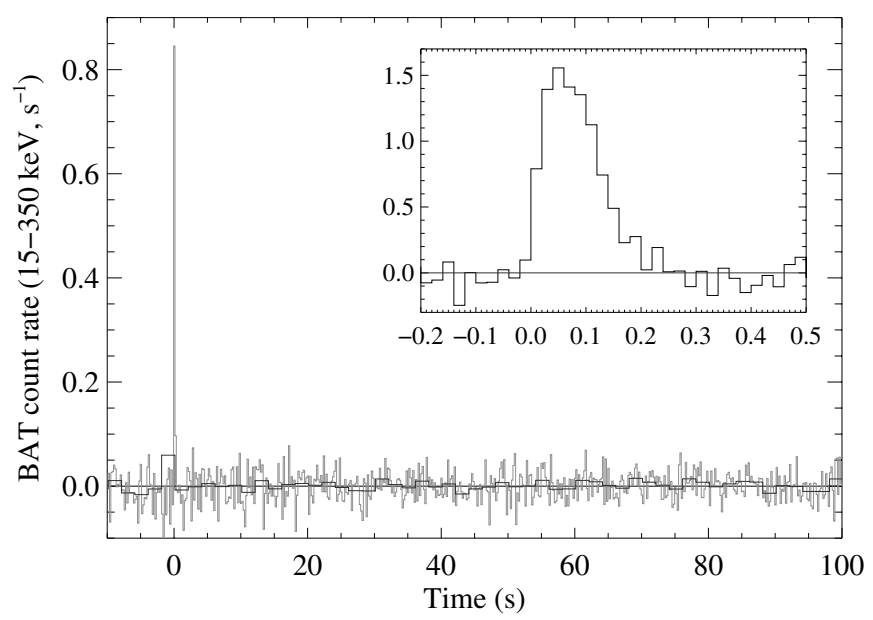

Figure 1. BAT light curve of GRB 100206A, taken from the sum of all four channels of data (15-350 keV) using the methods of Butler et al. (2007). The main plot shows the light curve binned at $2 \mathrm{~s}$ (dark gray) and $0.2 \mathrm{~s}$ (light gray); the inset shows the light curve binned at $0.02 \mathrm{~s}$. Times are referenced to $t=949498223.86 \mathrm{~s}$ (GPS). The burst is clearly short, with all detectable gamma-ray emission contained within a $0.25 \mathrm{~s}$ interval. There is no evidence of extended emission during the minutes after the trigger.

spectrum GRB with a faint, rapidly fading afterglow detected only in the X-ray band despite deep, early optical imaging. We also present observations of two galaxies in or near the X-ray error circle at a variety of wavelengths from optical through the mid-infrared. In Section 3, we analyze these data in further detail, measuring the metallicity and constraining the underlying stellar population of the brighter host candidate, which we show to be a luminous infrared galaxy (LIRG) at redshift $z=0.4068$ with a current SFR exceeding that of any previous short GRB by almost an order of magnitude. In Section 4, we argue that a posteriori statistical arguments strongly tie this galaxy to the short GRB, and examine the implications for the progenitor of the short-duration burst. While the current SFR is high, we note that the stellar mass is substantial and dominated by older stars, with no unambiguous evidence for starbursting, merging, or other short-lived features - consistent with an older progenitor, like that of other short-hard bursts. We summarize our conclusions in Section 5.

\section{OBSERVATIONS}

\subsection{Swift-BAT and Fermi}

GRB 100206A triggered the Burst Alert Telescope (BAT; Barthelmy et al. 2005) on the Swift satellite (Gehrels et al. 2004) at 13:30:05 on 2011 February 6 (UT dates are used throughout this paper). BAT data are automatically reduced by our automated pipeline using the methods of Butler et al. (2007). The BAT light curve (Figure 1) shows only a single spike starting at the trigger time and ending by $0.2 \mathrm{~s}$, with no evidence of extended emission (with $T_{90}=0.200 \pm 0.017 \mathrm{~s}$; Sakamoto et al. 2010).

The GRB also triggered the Gamma-ray Burst Monitor (Meegan et al. 2009) onboard the Fermi satellite. The Fermi light curve is similar to that seen by Swift - a single bright spike lasting $<0.2 \mathrm{~s}\left(T_{90}=0.13 \pm 0.05 \mathrm{~s}\right.$; Kienlin 2010). The spectrum of the burst is quite hard; the best-fit Band et al. (1993) model indicates $E_{\text {peak }}=439_{-60}^{+73} \mathrm{keV}$.

These properties place GRB 100206A unambiguously within the short-duration phenomenological class (see, for instance, Figure 1 of Levesque et al. 2010a). 

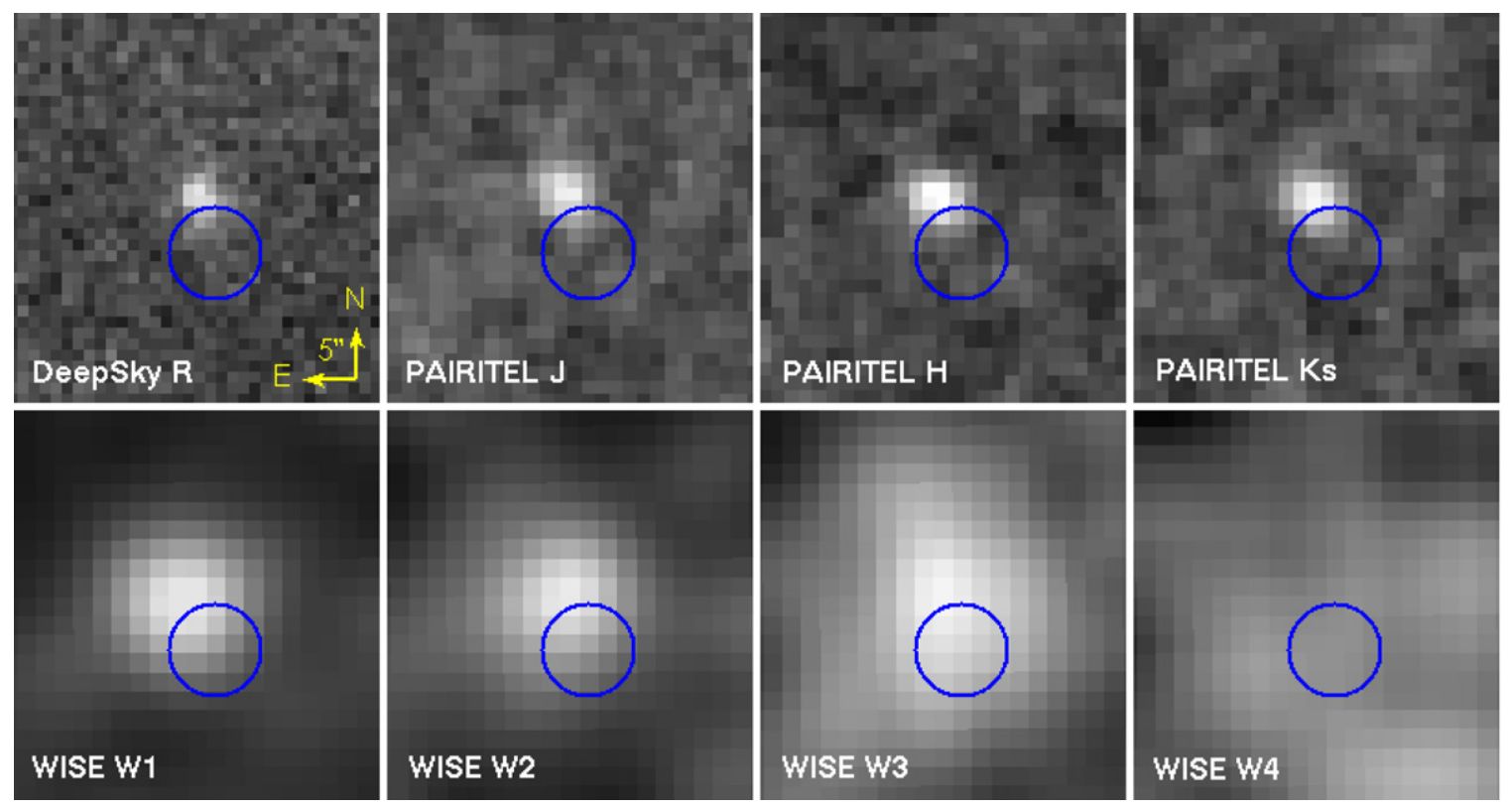

Figure 2. Unresolved images of the putative host galaxy (G1) of GRB 100206A in the optical and near-infrared (NIR) from the $1.2 \mathrm{~m}$ Palomar Oschin Telescope (from the Palomar/DeepSky project), the $1.3 \mathrm{~m}$ PAIRITEL, and the WISE all-sky mission. Although these telescopes are relatively insensitive to typical galaxies at cosmological distances, a bright source centered just outside the XRT error circle is well detected in every filter except W4 (22 $\mu \mathrm{m})$.

(A color version of this figure is available in the online journal.)

\subsection{Swift-XRT and UVOT}

Swift slewed immediately to the source and began pointed observations with the XRT (Burrows et al. 2005) at $74.6 \mathrm{~s}$ after the BAT trigger, followed by observations with the Ultraviolet Optical Telescope (UVOT; Roming et al. 2005) beginning at $78 \mathrm{~s}$. Only a faint and rapidly fading X-ray afterglow is seen, dropping from 0.2 to 0.01 counts $\mathrm{s}^{-1}$ during the first orbit; in total, only $24 \mathrm{X}$-ray photons were detected by the instrument during this observation. Swift made several return visits, none of which resulted in a detection above the background level.

During the first orbit, the event data are consistent with a light curve following a sharply falling, unbroken power law with decay index $\alpha=1.91_{-0.47}^{+0.40}$. Given the limited number of counts and large Galactic hydrogen column $\left(N_{\mathrm{H}} \approx 10^{21} \mathrm{~cm}^{-2}\right)$, no clear statements can be made about the intrinsic X-ray spectrum. The best available localization is the UVOT-enhanced XRT position (Goad et al. 2007): $\alpha=03^{\mathrm{h}} 08^{\mathrm{m}} 39^{\mathrm{s}} .03$ and $\delta=+13^{\circ} 09^{\prime} 25^{\prime \prime} \cdot 3$ (J2000, 3.'3 uncertainty at 90\% confidence).

No detection is reported in UVOT observations at any epoch. UVOT upper limits are given by Krimm et al. (2010). In addition, we stacked all available UVOT $u$-band imaging of this object to try to provide a deep limit on any host-galaxy emission; the integrated limit is $u>21.8 \mathrm{mag}$. Unfortunately, given the relatively large Galactic extinction in the field $\left(E_{B-V}=\right.$ 0.382 mag; Schlegel et al. 1998), this value is not particularly constraining.

\subsection{Ground-based Follow-up Observations}

GRB 100206A was observed by many different ground-based instruments within the first $24 \mathrm{hr}$ after the trigger (Bhattacharya et al. 2010; Guziy et al. 2010; Noda et al. 2010; Levan et al. 2010; Leloudas et al. 2010; Kuroda et al. 2010; Morgan et al. 2010; Yurkov et al. 2010; Mao et al. 2010; Berger et al. 2010; Nicuesa et al. 2010; Andreev et al. 2010; Rumyantsev et al. 2010). None of these observations report a detection of a varying source, although two static sources in the XRT error circle, initially noted by Miller et al. (2010) and Levan et al. (2010), will be discussed below. Given the faint X-ray afterglow, this nondetection of variability is unsurprising, as explained in further detail in Section 3.1.

\subsection{DeepSky}

We co-added 78 archival images from the DeepSky project at Palomar Observatory (Nugent et al. 2009) covering the field of GRB 100206A. The images were obtained in 2004-2008 from the Palomar-Quest Consortium at the Oschin Schmidt telescope. The limiting magnitude of the stack is $R \approx 23 \mathrm{mag}$. In the combined image, we detect a faint $(R=21.7 \pm 0.3$ mag relative to nearby USNO catalog stars) extended source, centered slightly outside the current XRT error circle to its northeast (Figure 2).

The random appearance of a source this bright close to a small $\mathrm{X}$-ray error circle is small $\left(P_{\text {chance }} \approx 0.03\right.$; see Section 4.1$)$, although not so small as to make the association unambiguous based on optical properties alone. However, this source has very unusual properties at other wavelengths that show it is far from being a typical galaxy.

\subsection{PAIRITEL}

The robotic Peters Automatic Infrared Imaging Telescope (PAIRITEL; Bloom et al. 2006) began observations of the GRB 100206 A field starting $13.1 \mathrm{hr}$ after the burst in the $J, H$, and $K_{s}$ filters simultaneously. No transient source was detected inside the error circle.

However, the host-galaxy candidate first seen in the DeepSky archival images was well detected in all three filters. PAIRITEL detection of galaxies at cosmological distances (Section 2.9) is unusual, leading us to first speculate that this galaxy may be an LIRG (Morgan et al. 2010). To improve the photometry of this source and verify the lack of fading behavior, we acquired additional imaging on 2011 October 20 and October 22, and combined data from all three epochs (total effective exposure 
Table 1

Photometry of Galaxies Inside the XRT Error Circle of GRB 100206A

\begin{tabular}{|c|c|c|c|c|c|c|}
\hline Instrument & $\begin{array}{c}\text { Exp. Time } \\
\text { (s) }\end{array}$ & Filter & $\begin{array}{r}\text { Magnitude }^{\mathrm{a}} \\
\text { G1 }\end{array}$ & $\mathrm{G} 1(z=0.41)$ & \multicolumn{2}{|c|}{$\mathrm{G} 2(z=0.80)$} \\
\hline Keck I/LRIS & 600 & $g$ & $23.71 \pm 0.17$ & 22.33 & $26.55 \pm 0.40$ & 25.17 \\
\hline Keck I/LRIS & 540 & $R$ & $21.34 \pm 0.09$ & 20.55 & $25.14 \pm 0.19$ & 24.34 \\
\hline Gemini-N/GMOS-N & 2400 & $i$ & $20.85 \pm 0.08$ & 20.09 & $24.69 \pm 0.05$ & 23.93 \\
\hline Gemini-N/GMOS-N & 1200 & $z$ & $20.21 \pm 0.05$ & 19.64 & $24.15 \pm 0.12$ & 23.57 \\
\hline PAIRITEL & 16333 & $J$ & $18.51 \pm 0.12$ & 19.06 & $>19.7$ & 20.3 \\
\hline PAIRITEL & 16333 & $H$ & $17.25 \pm 0.09$ & 18.41 & $>18.8$ & 19.9 \\
\hline PAIRITEL & 8143 & $K_{s}$ & $16.33 \pm 0.11$ & 18.03 & $>17.7$ & 19.4 \\
\hline WISE & & W1 & $15.74 \pm 0.06$ & 18.42 & & \\
\hline WISE & & $\mathrm{W} 2$ & $15.14 \pm 0.11$ & 18.47 & & \\
\hline WISE & & W3 & $11.23 \pm 0.16$ & 16.38 & & \\
\hline WISE & & W4 & $>8.58$ & $>15.18$ & & \\
\hline
\end{tabular}

Notes.

a Observed value, not corrected for Galactic extinction.

${ }^{\mathrm{b}}$ Corrected for Galactic extinction ( $\left.E_{B-V}=0.38 \mathrm{mag}\right)$.

time of $4.34 \mathrm{hr}$ ) with the exception of about $2 \mathrm{hr}$ of $K_{s}$-band data which was not usable due to poor sky conditions.

Aperture photometry was performed using custom Python software, utilizing Source Extractor (SExtractor; Bertin \& Arnouts 1996) as a back end. Calibration was performed by determining the zero point for each image by comparison to Two Micron All Sky Survey (Skrutskie et al. 2006) magnitudes using 19 calibration stars. As the galaxy is clearly extended in higher-resolution images, we employ a large aperture radius of $3^{\prime \prime}$.

Indeed, no evidence of fading (to limits of $J>19.8$, $H>19.3$, and $K_{s}>16.8 \mathrm{mag}$ ) is observed between the initial epoch and the late-time co-add (the $K_{s}$-band constraint is poor due to the low quality of the second-epoch image). Photometry of the two galaxies using the final, combined stacks is presented in Table 1.

\subsection{Gemini Imaging}

Two epochs of deep imaging were acquired with the Gemini Multi-Object Spectrometer (GMOS-N) on Gemini-North. In the first epoch (starting at 05:34, $14 \mathrm{hr}$ after the trigger), five dithered exposures of $240 \mathrm{~s}$ each were obtained in the $z$ band, immediately followed by a similar sequence of $5 \times 240 \mathrm{~s}$ exposures in the $i$ band. The $i$-band sequence was repeated five days later, between 06:41 and 07:05 on 2010 February 12. Conditions were excellent during both observations, with an average seeing of about 0.8 .

The bright archival galaxy first seen in our DeepSky image is resolved by these observations into an extended disk of about 5 " diameter (Figure 3). We denote this galaxy "G1" in the remainder of the paper. A second, much fainter point-like source (which we denote "G2") is also seen $\sim 8$ " to the south of this object.

Berger et al. (2010) performed image subtraction between the two epochs of $i$-band imaging and reported no variation either between these epochs or in comparison with a William Herschel Telescope image taken $7 \mathrm{hr}$ after the trigger-including, in particular, no variation of the point-like source G2 which had been initially suggested by Levan et al. (2010) as a candidate afterglow. (Indeed, our spectroscopy verifies that this object is a background galaxy; Section 2.9.)

We downloaded all Gemini frames from the Gemini Science archive and reduced them independently using the GMOS IRAF

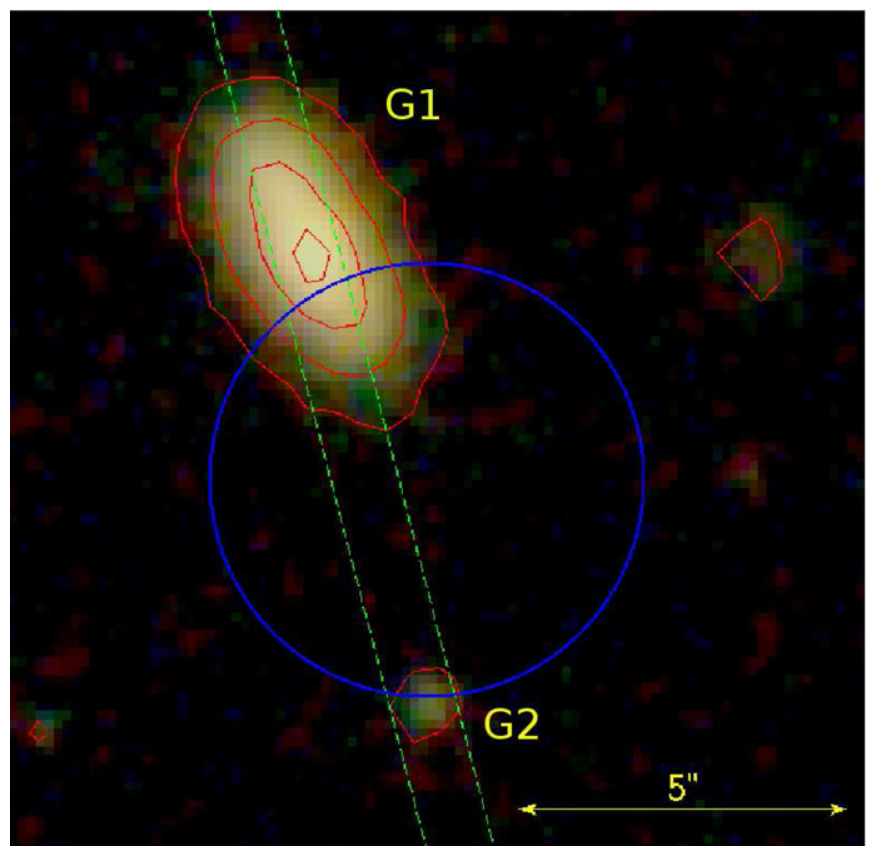

Figure 3. Deep imaging of the field of GRB 100206A from the Keck $10 \mathrm{~m}$ telescope and Gemini-North $8 \mathrm{~m}$ telescope, combined into a false-color image using the $R, i$, and $z$ filters. The bright galaxy G1 resolves into a highly inclined disk, with most of the southern half of the galaxy enclosed by the XRT error circle (shown in blue). A second, much more compact galaxy is also evident, centered at the southern edge of the XRT error circle, which we label G2. Isophotes of G1 show a subtle asymmetry that probably results from additional substructure within the galaxy (see also Figure 8.) The dashed green line indicates the position of the LRIS slit during our long-slit spectroscopy. North is up and east is left.

(A color version of this figure is available in the online journal.)

reduction tools. We first co-added the data obtained during each of the two separate $i$-band epochs, and subtracted the two images using HotPants, ${ }^{7}$ confirming the lack of variability reported by Berger et al. ${ }^{8}$ We measure an improved $5 \sigma$ upper limit of $i>25.9$ mag for any point source varying between the two frames over an 0 '. 8 aperture within the XRT error circle.

\footnotetext{
7 http://www.astro.washington.edu/users/becker/hotpants.html

8 One marginal source does appear with a nominal significance of about $5 \sigma$ at $\alpha=03^{\mathrm{h}} 08^{\mathrm{m}} 38^{\mathrm{s}} .846$ and $\delta=+13^{\circ} 09^{\prime} 24^{\prime \prime} .25$ (J2000) but it seems to be mostly due to a weak negative artifact in the late-time reference image rather than the detection of positive variation in the first image.
} 


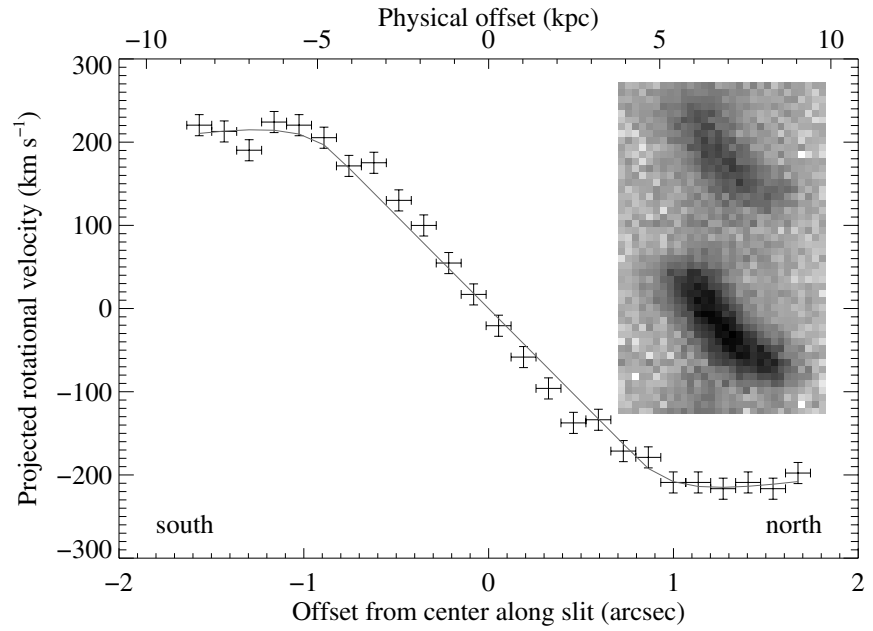

Figure 4. Projected rotation curve for $\mathrm{G} 1$, determined from $\mathrm{H} \alpha$ and $\left[\mathrm{N}_{\mathrm{II}}\right]$ emission lines. Note that the slit is $1^{\prime \prime}(5.4 \mathrm{kpc})$ wide and at a (slightly) different orientation than the major axis of the galaxy, so the true major-axis rotation velocities of the galaxy are likely to be somewhat larger than the values indicated here. (The galaxy itself is also likely inclined $\sim 20^{\circ}$ from edge-on; Figure 8 .) The relevant region of the two-dimensional spectrum is shown in the inset. The gray line shows the rotation curve expected from a simple mass model (a singular isothermal sphere with a constant density center) with a total dynamical mass of $\sim 1.7 \times 10^{11} M_{\odot}$.

Given the lack of variation and comparable, good conditions over the two nights, we then stacked all $10 i$-band exposures over both epochs to produce a single, deep image. The five $z$-band frames from the first night were also co-added into a separate $z$-band stack. Using secondary standard stars from our P60 calibration of the field (Section 2.8), we performed aperture photometry of G1 using a radius of $3^{\prime \prime}$. Photometry of G2 was calculated using the same standards, using a $1^{\prime \prime}$ radius aperture; see Table 1.

\subsection{Keck Imaging}

To obtain additional color information, on the night of 2010 August 2 we imaged the field of GRB 100206A with the Low Resolution Imaging Spectrograph (LRIS; Oke et al. 1995) on the $10 \mathrm{~m}$ Keck I telescope. Using the D560 dichroic, we acquired images in the $g$ and $R$ filters simultaneously, with $3 \times 200 \mathrm{~s}$ integration in $g$ and $3 \times 180 \mathrm{~s}$ integration in $R$. Reduction was accomplished using a custom LRIS imaging pipeline. As with the Gemini images, we performed aperture photometry of the two possible host galaxies G1 and G2 using $3^{\prime \prime}$ and $1^{\prime \prime}$ radius apertures, respectively, using our secondary field standards. Again, the photometry is reported in Table 1.

\subsection{P60 and Nickel Photometric Calibrations}

To calibrate the optical photometry, we acquired independent calibrations using the roboticized 60 inch telescope at Palomar Observatory (P60; Cenko et al. 2006) and the Nickel $1 \mathrm{~m}$ reflector at Lick Observatory, both on 2011 August 9. Numerous standard fields from Landolt (2009) were observed throughout the night in the gRiz filters (P60) or BVRI filters (Nickel). The Nickel photometry was then transformed to giz using the transformation equations of Jester et al. (2005). The two calibrations show good consistency, with $<0.04$ mag systematic differences in each of the Riz filters for bright stars. A slightly larger offset of $0.1 \mathrm{mag}$ is observed in the $g$ band. To calibrate the galaxy photometry, we take the average of the two $g-, R$-, and $i$-band calibrations, adding a small calibration component to the uncertainty. Only the P60 data are used to calibrate the $z$ band. Final, calibrated photometry of the two galaxies near the XRT error circle is presented in Table 1.

\subsection{Keck Spectroscopy}

We acquired spectra using LRIS on the Keck I 10 m telescope on two occasions, both using the D560 dichroic, the 600/4000 grism, the 400/8500 grating, and a $1^{\prime \prime}$ slit. The first epoch was obtained on 2010 February 7 between 06:17 and 06:40 UT, shortly after the burst. Two exposures, each of duration $600 \mathrm{~s}$ (on the red side; slightly longer on the blue side), were acquired at a slit position angle (P.A.) of 18.2 between 06:17 and 06:39. (LRIS is equipped with an Atmospheric Dispersion Corrector (Phillips et al. 2006), enabling us to observe away from the parallactic angle without slit losses.) Seeing conditions were poor $\left(\sim 1^{\prime \prime} .5\right)$ throughout the integration. Nevertheless, the galaxy was well detected in the red part of the spectrum, showing strong emission lines of $\mathrm{H} \alpha$ and [N II], as we previously noted (Cenko et al. 2010). The slit orientation was fortuitously close to the galaxy major axis and these lines show clear rotational structure.

A second epoch was acquired on 2011 August 2, shortly following our imaging that same night (Section 2.7). A slit P.A. of 14.5 was used to simultaneously cover both G1 and G2 at the same time; one exposure was acquired of $900 \mathrm{~s}$ duration (on the red side; the integration on the blue side lasted an additional $10 \mathrm{~s}$ ). This P.A. is still close to the major axis of the galaxy (Figure 3$)$. Conditions were excellent ( 0 '.8 seeing) and the spectrum is of significantly higher quality than the one obtained during the earlier epoch, showing both lines of the [N II] doublet as well as [S II], $\mathrm{H} \beta$, and [O II]. Accordingly, only this spectrum is used in our final analysis.

The spectra were reduced using custom reduction software written in IDL. The traces of the two galaxies were extracted separately and flux-calibrated relative to spectroscopic standards BD28+4211 (blue; Oke 1990) and BD17+4708 (red; Oke \& Gunn 1983) and then normalized by performing synthetic photometry in the $g$ (blue), $R$, and $i$ bands, and re-scaling each side to match the broadband photometry measured in our imaging. Both objects are observed to have multiple strong emission lines, identifying them as galaxies at redshifts of $z=0.4068(\mathrm{G} 1)$ and $z=0.803(\mathrm{G} 2)$. In the case of $\mathrm{G} 1$, strong rotational "shearing" is seen in the two-dimensional spectrum $\left(v / c=420 \mathrm{~km} \mathrm{~s}^{-1}\right.$ between opposite ends of the galaxy, corresponding to $13 \AA$ in the vicinity of $\mathrm{H} \alpha$ ), so a simple extraction would produce much poorer resolution than what is provided by the instrument. To remove this shear, we calculate the rotation curve of the galaxy (plotted in Figure 4) by finding the maximum of the cross-correlation function along each row of the trace relative to the galaxy center in the region of the strong lines of the $\mathrm{H} \alpha-[\mathrm{N}$ II] complex, and then resample each line (across the full trace) by the appropriate amount to remove the systematic rotation. This procedure allows us to achieve a resolution comparable to the instrumental resolution of $\sim 7 \AA$. Following this step, the spectrum is extracted normally using a window of 3 ".24 (diameter). (The emission lines of G2 are not resolved, and this spectrum is extracted normally with no additional steps.) The final one-dimensional spectra of G1 and G2 are presented in Figures 5 and 6, respectively.

\subsection{WISE Archival Observations}

We searched the Wide-field Infrared Survey Explorer (WISE; Wright et al. 2010) archive for observations at the position of 


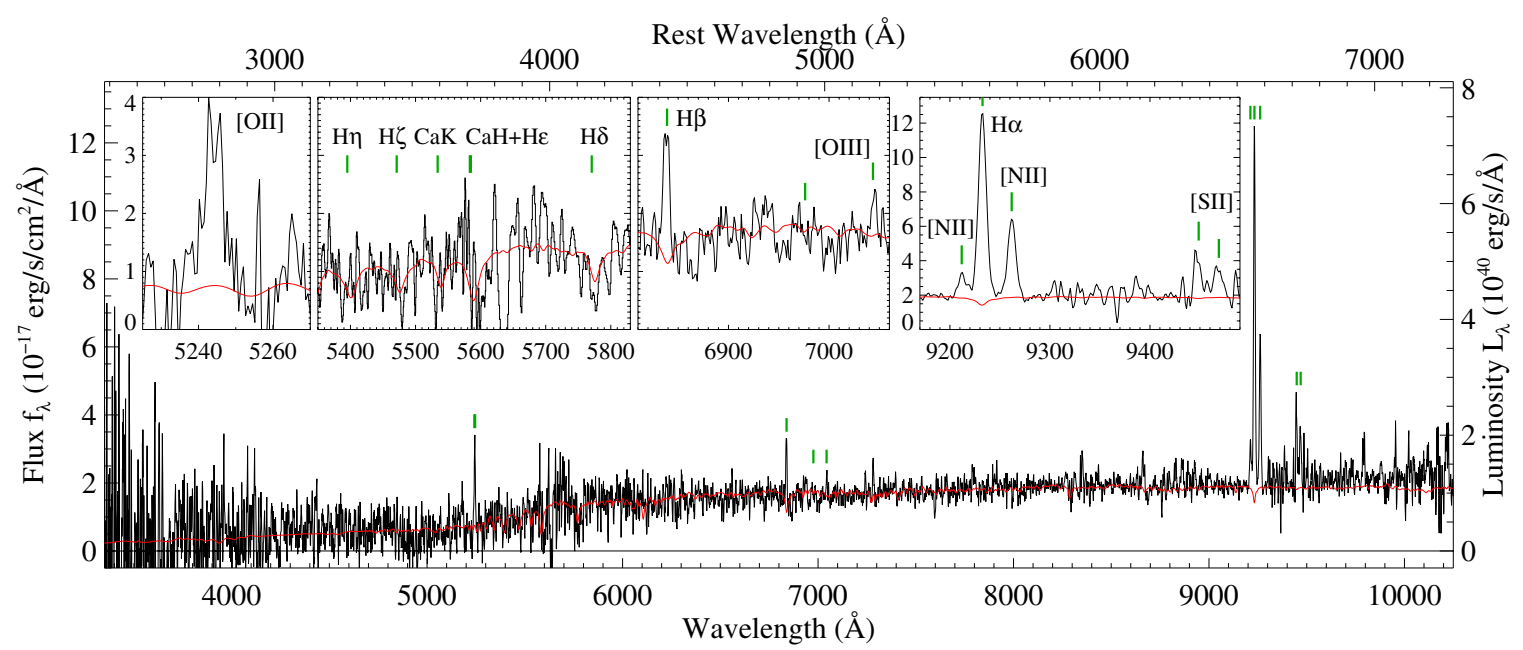

Figure 5. Spectroscopy of G1, the putative host galaxy. The combined LRIS blue+red spectrum is shown in the main panel, with the positions of detected emission lines indicated. In addition, the red curve shows the best stellar-continuum model from a fit to our broadband (and synthetic narrowband) photometry. The small deviation between this model and the observations at $>9500 \AA$ is probably due to uncertainties in the spectrophotometry in the long-wavelength region. At top, the insets show regions around specific lines, including [O II], the Balmer absorption lines, $\mathrm{H} \beta+\left[\mathrm{O}\right.$ III], and $\mathrm{H} \alpha+[\mathrm{N}$ II $]+\left[\mathrm{S}_{\mathrm{II}}\right]$.

(A color version of this figure is available in the online journal.)

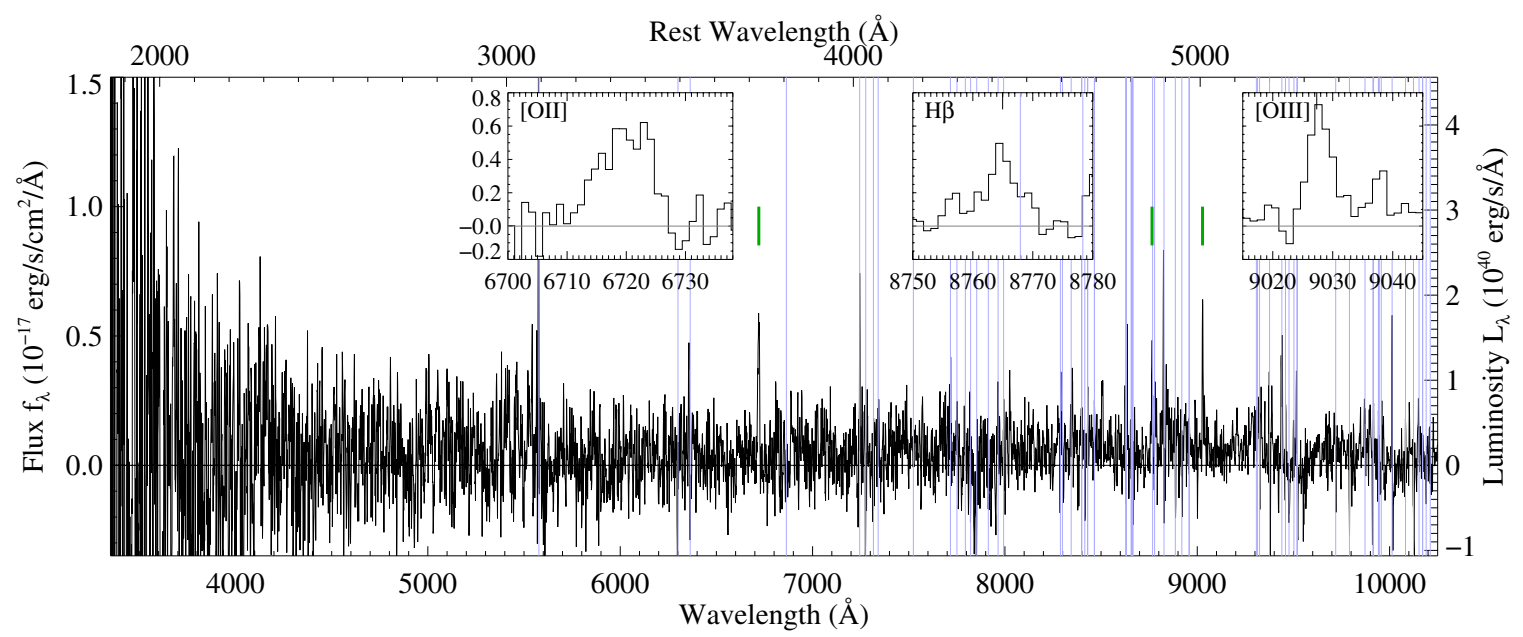

Figure 6. Spectrum of G2, the faint galaxy at the south end of the XRT error circle. Secure detection of the [O II] and [O III] lines show this to be a star-forming galaxy at $z=0.803$. The vertical gray lines indicate the centers of strong sky emission lines.

(A color version of this figure is available in the online journal.)

this GRB. The field was covered by the WISE preliminary data release, and a source is clearly detected at the position of G1 in both the archival images and in the photometric catalog in filters W1-W3 (no detection is evident in W4), as shown in Figure 2. In Table 1, we present catalog magnitudes of this object from the archive.

\section{ANALYSIS}

\subsection{Afterglow and Kilonova Models}

Despite extensive optical and NIR follow-up observations during the first night (including some data on timescales as short as minutes and a very deep image with Gemini), no afterglow was detected from this event. Using standard assumptions about the intrinsic spectral index of a GRB afterglow, we examined whether these limits might usefully constrain the properties of the GRB or the extinction column.

In Figure 7, we plot the "light curve" of the X-ray afterglow (consisting of two binned detections followed by upper limits) and scale all reported UV, optical, or NIR upper limits from the
GCN Circulars to the X-ray band (1 keV equivalent photon energy) based on an assumed afterglow spectral index of $\beta_{\mathrm{OX}}=0.5$, the minimum value expected in the synchrotron afterglow model (Jakobsson et al. 2004; a value below this line would indicate a "dark" burst). Any upper limits lying below the X-ray light curve are inconsistent with this basic model and would require either an unusually blue/hard intrinsic spectral index or extinction within the host galaxy to suppress the optical flux. As is evident from the figure, none of the limits constrain the afterglow in this way. In fact, given that the observations after $10^{4} \mathrm{~s}$ are upper limits and the light curve could have steepened even if we assume a much softer/redder spectral index (up to $\beta_{\mathrm{OX}}=1.1$, which corresponds to an unbroken spectral index between the X-ray and optical given typical X-ray spectral indices and represents the maximum value expected in the synchrotron model), the optical photometry still imposes no constraint on any additional host extinction.

The time of the deep Gemini limit is better timed to constrain emission from a Li \& Paczyński (1998) mini-supernova or "kilonova" (see also Kulkarni 2005; Metzger et al. 2010), which 


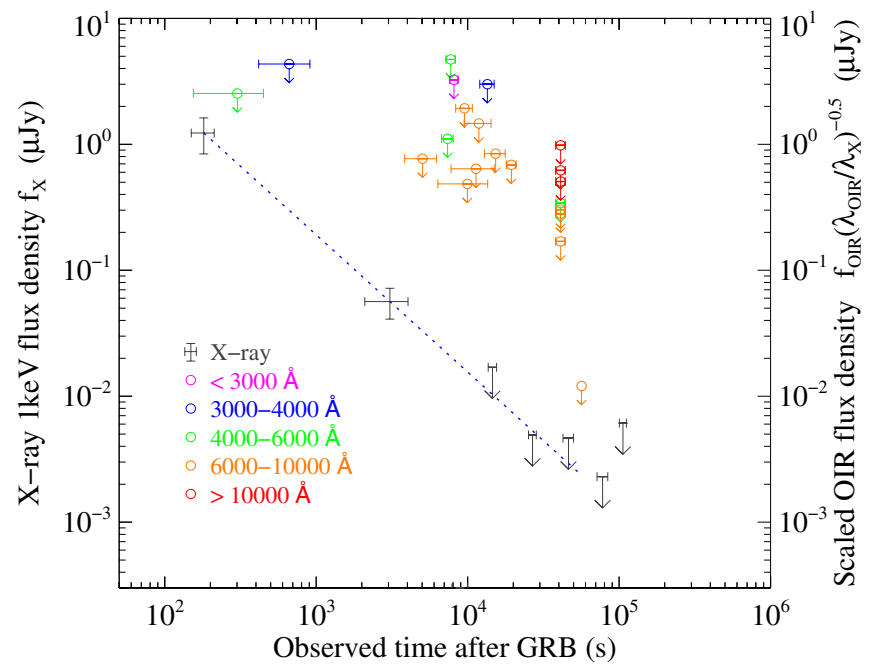

Figure 7. X-ray and UV/optical/NIR observations of GRB 100206A. The $\mathrm{X}$-ray light curve is plotted as flux density $\left(f_{v}\right)$ at $1 \mathrm{keV}$. To meaningfully place the UV/optical/NIR observations (all of which are upper limits) on the same plot, we calculate the flux corrected for Galactic extinction and extrapolate it into the X-ray band assuming $\beta=0.5\left(f_{v}=v^{-\beta}\right)$. Given the extremely faint $\mathrm{X}$-ray afterglow, none of the optical limits is constraining.

(A color version of this figure is available in the online journal.)

is expected to peak at $t \approx 1$ day. After correcting for Galactic extinction, the GMOS $i$-band limit corresponds to a specific luminosity limit of $v L_{v}<10^{42} \mathrm{erg} \mathrm{s}^{-1}$ at $t=11 \mathrm{hr}$, if the GRB is at the redshift of the brighter galaxy $(z=0.4068)$ and is unextinguished by dust within its host. This is comparable to the limits presented by Kocevski et al. (2010) and broadly rules out models with large energy conversion factors and ejecta masses $\left(f \gtrsim 10^{-5}\right.$ and $\left.M \gtrsim 10^{-2.5} M_{\odot}\right)$, but is significantly less constraining than the limits presented on radioactivepowered emission from GRB 050509B by Hjorth et al. (2005). Furthermore, even our weak limit is subject to the strong caveat that the extinction within this host galaxy appears to be quite large (Section 3.6) and the assumption of no extinction may not be valid.

\subsection{Morphology}

The galaxy G1 has a disk-like morphology (Figure 3) and is seen at a high inclination. It is also quite large, with significant emission extending out $1^{\prime \prime} .5(8 \mathrm{kpc})$ in each direction away from the nucleus. Some subtle asymmetry is visible: the location of maximum flux is displaced by about one pixel $\left(00^{\prime} .15\right)$ from the center of the outer isophotes, and there is a hint of displacement between the northern and southern sides of the disk from the nucleus, possibly suggestive of spiral or bar structure.

To gain further insight into the structure of the galaxy, we used the software package GALFIT (Peng et al. 2002) to model the system as an inclined Sérsic (1963) disk. Our modeling is performed using the Gemini GMOS $i$-band image, which has the best signal-to-noise ratio and seeing quality of the filters used. A bright, isolated, nonsaturated, nearby star in the image is used as the point-spread function (PSF) model. Because a simple Sérsic fit gives significant residuals (as expected, given the visual asymmetry), we extend this basic model by successively adding individual Gaussians initially centered at locations of positive residuals and iteratively repeat the fit until no residuals are evident above the background noise. We found three such components to be needed within the physical extent of the disk: one each on either side of the nucleus in the disk plane, each at a displacement of 1".3 (7 kpc in projection), plus an additional component displaced 1 .". 1 east $(6 \mathrm{kpc})$ of the nucleus. (A fourth component 3 .'2 northeast of the galaxy is also required by the fitting procedure, but is probably not related to the system.) These additional components combined only contribute $\sim 20 \%$ of the total flux of the system, which is still dominated by the Sérsic disk.

Whether or not these additional parameters are added, similar results for the Sérsic parameters are achieved (Sérsic index $n=$ 0.58 and axis ratio $b / a=0.31$ for a fit with the disk only and no extra components; $n=1.27, b / a=0.40$ for the final model with four extra components). Evidently, the stellar light in this system follows a nearly exponential disk ( $n=1$; typical for disk galaxies), inclined at an angle of $\sim 70^{\circ}$ from face-on.

The decomposition described above also functions as a basic deconvolution of the image near the galaxy. In Figure 8, we show both the observed $i$-band image and its deconvolved equivalent, which is the sum of the model produced by GALFIT (not reconvolved with the PSF) and the fit residuals. The residuals are added both to accurately show the noise level, and also to avoid falsely removing any real signal that may be left in the residuals.

Deconvolution should always be approached with caution, and our modeling cannot identify faint, small-scale $\left(\ll 1^{\prime \prime}\right)$ structures that are likely to be present or more complex structures such as bars or arcs. Nevertheless, since the observed components are all separated on scales larger than the $0^{\prime \prime} .8$ seeing disk, we expect that they likely represent real substructure in the galaxy-projected spiral arms or localized intense star-forming regions (the regular $\mathrm{H} \alpha$ rotation curve in Figure 4 suggests they are not merging galaxies). Further observations with better image quality (such as from the Hubble Space Telescope) would be necessary to unambiguously resolve the structure of this system. Nevertheless, it is clear that G1 is a morphologically complex but predominately disk-like galaxy.

\subsection{Emission-line Measurements}

The clear detections of numerous, bright emission lines in the spectrum of G1 allow us to apply standard diagnostics of the SFR, extinction, and metallicity to this galaxy.

We first extract the emission-line fluxes by assuming a flat continuum with no underlying stellar absorption component. Taking a small region around each emission line or line complex, we fit a Gaussian function (or, in the case of blended lines, several summed Gaussians) added to a linear component representing the underlying flux with the mpfit package within IDL. For each line, the integrated flux and uncertainty are obtained from the fit. The results are presented in the third column of Table 2.

While the strong $\mathrm{H} \alpha$ line (combined with the modest $[\mathrm{N} \mathrm{II}] / \mathrm{H} \alpha$ and $[\mathrm{O} \mathrm{III}] / \mathrm{H} \beta$ ratios which associate this line with star formation rather than active galactic nucleus activity; Baldwin et al. 1981) gives unambiguous evidence of rapid, recent star formation, several other indicators suggest that an older stellar component is also present. Specifically, while many of the higher-order Balmer lines fall in the wavelength region of the dichroic where sensitivity is relatively poor $(\sim 5550-5790 \AA)$, we do see a dip at the location of $\mathrm{H} \delta$ and lower-significance dips at the locations of $\mathrm{H} \eta$ and $\mathrm{H} \zeta$, as well as (possibly) $\mathrm{Ca}$ $\mathrm{H} \& \mathrm{~K}$ (inset of Figure 5). Interestingly, we see neither emission nor absorption at the location of $\mathrm{H} \gamma$ where sensitivity is good, suggesting that the emission and absorption are of comparable strength, resulting in a flat apparent spectrum (the instrument 


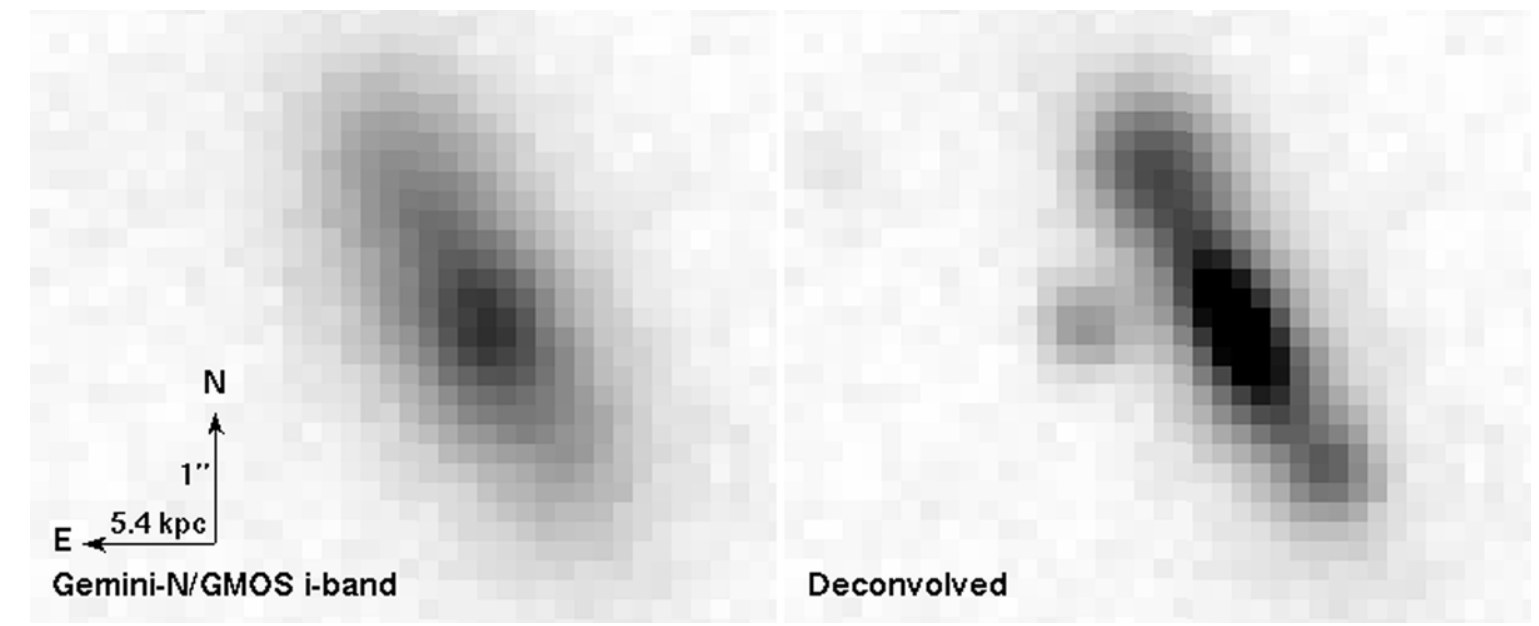

Figure 8. Field of GRB 100206A imaged in the $i$ band using the Gemini-North $8 \mathrm{~m}$ telescope, with no additional processing (left) and as deconvolved by our GALFIT model (right). In addition to a Sérsic exponential disk, three other compact components are required to obtain a fit without residuals: two along the major axis displaced $7 \mathrm{kpc}$ in projection from the center and an additional object close to the minor axis.

Table 2

Fluxes of Detected Emission Lines in G1 and G2

\begin{tabular}{|c|c|c|c|c|}
\hline Galaxy & Line Species & $\begin{array}{c}\text { Rest Wavelength } \\
\text { (§) }\end{array}$ & $\begin{array}{c}\text { Unsubtracted Flux }^{\mathrm{a}} \\
\left(10^{-17} \mathrm{erg} \mathrm{s}^{-1}\right)\end{array}$ & $\begin{array}{c}\text { Subtracted Flux } \\
\left(10^{-17} \mathrm{erg} \mathrm{s}^{-1}\right)\end{array}$ \\
\hline \multirow[t]{8}{*}{ G1 } & {$[\mathrm{O} \mathrm{II}]^{\mathrm{c}}$} & 3727 & $16.2 \pm 1.8$ & $19.6 \pm 2.1$ \\
\hline & $\mathrm{H} \beta$ & 4861.33 & $12.7 \pm 1.8$ & $21.8 \pm 1.9$ \\
\hline & [O III] & 5006.84 & $5.9 \pm 1.5$ & $5.1 \pm 1.2$ \\
\hline & $\mathrm{H} \alpha$ & 6562.82 & $108.6 \pm 1.4$ & $112.8 \pm 1.6$ \\
\hline & {$[\mathrm{N}$ II] } & 6548.06 & $12.8 \pm 1.1$ & $13.1 \pm 1.2$ \\
\hline & {$\left[\mathrm{N}_{\mathrm{II}}\right]$} & 6583.57 & $46.4 \pm 1.2$ & $45.7 \pm 1.3$ \\
\hline & {$[\mathrm{S}$ II $]$} & 6716.44 & $20.4 \pm 3.2$ & $20.4 \pm 3.1$ \\
\hline & [S II] & 6730.82 & $14.1 \pm 1.8$ & $13.6 \pm 1.7$ \\
\hline \multirow[t]{3}{*}{$\mathrm{G} 2$} & {$[\mathrm{OII}]^{\mathrm{c}}$} & 3727 & $6.0 \pm 0.8$ & \\
\hline & $\mathrm{H} \beta$ & 4861.33 & $2.8 \pm 1.7$ & \\
\hline & [O III] & 5006.84 & $3.1 \pm 0.4$ & $\cdots$ \\
\hline
\end{tabular}

\section{Notes.}

a Assuming a flat continuum, corrected for Galactic extinction.

b After subtracting the model continuum, corrected for Galactic extinction.

${ }^{c}$ Combined flux of both lines of the [O II] doublet.

resolution of $7 \AA$ is comparable to the intrinsic width of the Balmer line). We also see evidence for a strong Balmer break in the photometry (Section 3.6).

The presence of Balmer absorption features raises the prospect that the simple procedure above used to measure the emission-line fluxes may be systematically underestimating some line strengths. The large equivalent width of $\mathrm{H} \alpha$ suggests that it is unlikely to be affected by uncertainties in the underlying continuum by more than a few percent, but the weaker $\mathrm{H} \beta$ line may be much more strongly affected, which would impact the spectroscopically derived extinction (and therefore the extinction-corrected SFR). Consequently, after deriving an estimate of the stellar continuum from population synthesis modeling (Section 3.6, below), we subtract the continuum flux given by that model and then repeat the line fits with the continuum removed. The results are given in the fourth column of Table 2 .

For galaxy G2, we only present the line fluxes using the basic linear continuum-subtraction model. As the continuum trace of this galaxy is extremely weak, this is a good assumption at the level of uncertainty in our measurements. The fluxes of the probable lines (note that $\mathrm{H} \beta$ is blended with a strong night-sky line and not clearly present) are given in Table 2 .

\subsection{Chemical Abundance Analysis}

The strong and unambiguous detection of numerous emission lines from G1 makes it possible to measure the metallicity of the galaxy; this has been successfully done for only a few shortGRB hosts so far (e.g., Berger 2009; Rowlinson et al. 2010), although long-GRB and core-collapse supernova metallicities have been studied extensively (e.g., Modjaz et al. 2008; Savaglio et al. 2009; Levesque et al. 2010b). Metallicity is expected to influence not only the lives of stars but also the outcome of their deaths as different kinds of explosion; thus, the burgeoning field of metallicity studies for different kinds of explosive events from both observational (for a review, see Modjaz 2011) and theoretical (e.g., Hirschi et al. 2005; Yoon \& Langer 2005; Fryer et al. 2007) perspectives has emerged over the last few years.

The nebular oxygen abundance (usually reported in units of $12+\log (\mathrm{O} / \mathrm{H}))$ is the canonical choice of metallicity indicator for studies of the interstellar medium. Using the continuum-corrected line fluxes from Table 2 and correcting for internal reddening $E_{B-V}=0.60 \pm 0.42$ mag assuming $\mathrm{H} \alpha$ / $\mathrm{H} \beta=2.86$ from Case B recombination (Osterbrock 1989), we measure the nebular oxygen abundance of the galaxy following the technique described by Modjaz et al. $(2008,2011)$ and references therein. Results are summarized in Table 3. Using the scales from Pettini \& Pagel (2004, PP04), we find 12 $+\log (\mathrm{O} / \mathrm{H})=8.74_{-0.03}^{+0.02}$ based on the $[\mathrm{N} \mathrm{II}] / \mathrm{H} \alpha$ diagnostic $(\mathrm{PP} 04-\mathrm{N} 2)$, and $12+\log (\mathrm{O} / \mathrm{H})=8.81_{-0.12}^{+0.10}$ based on the [O III]/[N II] (PP04-O3N2) prescription. Likewise, we find 12 $+\log (\mathrm{O} / \mathrm{H})=9.17_{-0.11}^{+0.09}$ on the scale of Kewley \& Dopita (2002, $\mathrm{KD} 02)$ and $12+\log (\mathrm{O} / \mathrm{H})=8.82_{-0.16}^{+0.11}$ on the scale of McGaugh (1991, M91). Here, we have computed the uncertainties in the measured metallicities by explicitly including the statistical uncertainties of the line-flux measurements, a conservative estimate of the uncertainty arising from continuum absorption contamination, and those in the derived galaxy reddening, and we propagate them into the metallicity determination. The ionization parameter $q$, iteratively derived in the KD02 models, is $\log _{10}(q)=7.59_{-0.27}^{+0.4}$.

Given the agreement of the $T_{e}$-based metallicity scale (which the PP04-O3N2 scale is close to) with metallicity measurements of stars (Bresolin et al. 2009), we conclude that the true value of the gas-phase oxygen abundance is most likely to be closer to the lower end of the estimated range, approximately $12+$ 
Table 3

G1 Metallicity Determinations ${ }^{\mathrm{a}}$

\begin{tabular}{|c|c|c|c|c|}
\hline Method & Type $^{\text {b }}$ & Relevant Lines ${ }^{c}$ & $12+\log (\mathrm{O} / \mathrm{H})^{\mathrm{d}}$ & $Z / Z_{\odot}^{\mathrm{e}}$ \\
\hline $\mathrm{PP} 04-\mathrm{N} 2$ & Empirical & {$[\mathrm{N}$ II $], \mathrm{H} \alpha$} & $8.74_{-0.03}^{+0.02}$ & $1.12_{-0.07}^{+0.05}$ \\
\hline $\mathrm{PP} 04-\mathrm{O} 3 \mathrm{~N} 2$ & Empirical & {$[\mathrm{O} \mathrm{III}], \mathrm{H} \beta,[\mathrm{N} \mathrm{II}], \mathrm{H} \alpha$} & $8.81_{-0.12}^{+0.10}$ & $1.32_{-0.32}^{+0.34}$ \\
\hline $\operatorname{M91}\left(R_{23}\right)$ & Theoretical & {$[\mathrm{O} \mathrm{II}],[\mathrm{O} \mathrm{III}], \mathrm{H} \beta$} & $8.82_{-0.16}^{+0.11}$ & $1.35_{-0.30}^{+0.87}$ \\
\hline KD02 (combined) & Theoretical & {$\left[\mathrm{O}_{\mathrm{II}}\right],\left[\mathrm{O}_{\mathrm{III}}\right],[\mathrm{N}$ II $],\left[\mathrm{S}_{\mathrm{II}}\right], \mathrm{H} \beta$} & $9.17_{-0.11}^{+0.09}$ & $3.02_{-0.93}^{+0.87}$ \\
\hline Fitted $^{\mathrm{f}}$ & Theoretical & {$[\mathrm{O}$ II $],[\mathrm{O}$ III $],[\mathrm{N}$ II $],[\mathrm{S} \mathrm{II}], \mathrm{H} \alpha, \mathrm{H} \beta$} & $9.04 \pm 0.09$ & $2.25 \pm 0.4$ \\
\hline
\end{tabular}

Notes.

a Integrated values for G1. Spatially resolved measurements for the southern component are given in Section 3.5.

b Principle of the metallicity calibration: theoretical (using photoionizaion models) or empirical (calibrated to observations of local star-forming regions).

${ }^{\mathrm{c}}$ Lines directly used as part of the metallicity determination prescription. Most models additionally include $\mathrm{H} \alpha$ and $\mathrm{H} \beta$ to determine the host extinction, which is then used to correct the metal line fluxes.

${ }^{\mathrm{d}}$ Gas-phase oxygen abundance.

e Oxygen Abundance in solar units as inferred from the previous column, assuming a solar value of $\log [\mathrm{O} / \mathrm{H}]_{\odot}=8.69$.

${ }^{\mathrm{f}}$ Weighted average of the lower three models in Table 5; see Section 3.6 for details.

$\log (\mathrm{O} / \mathrm{H})=8.8$. Based on the most recent estimate of the solar oxygen abundance $(12+\log (\mathrm{O} / \mathrm{H})=8.69$; Asplund et al. 2009), this would indicate a mildly super-solar value $\left(Z=1.1 Z_{\odot}\right)$. However, most previous metallicity work on short-GRB host galaxies (e.g., Berger 2009) has used the KD02 scale exclusively, which tends to produce systematically higher values than PP04 (see detailed discussions in Kewley \& Ellison 2008; Moustakas et al. 2010 and references for possible reasons for the systematic offsets between different abundance diagnostics). For more direct comparison with known GRB host galaxies, then, the KD02 value of $12+\log (\mathrm{O} / \mathrm{H})=9.17_{-0.11}^{+0.09}$ $\left(Z=3 Z_{\odot}\right)$ is more informative.

In either case, G1 (if it is indeed the host of GRB 100206A; Section 4.1) would be the most metal-rich host galaxy of any GRB (long or short) to date. To our knowledge, the only longGRB host galaxies with super-solar metallicity (as determined via emission spectroscopy, via the KD02 scale ${ }^{9}$ ) are the hosts of GRB $020819(12+\log (\mathrm{O} / \mathrm{H})=9.0 \pm 0.1$; Levesque et al. 2010c), GRB $050826(12+\log (\mathrm{O} / \mathrm{H})=8.83 \pm 0.1$; Levesque et al. 2010b), and GRB $051022(12+\log (\mathrm{O} / \mathrm{H}) \approx 8.77$; Graham et al. 2010, 2011). Short-GRB host galaxies with potentially super-solar metallicities include the hosts of GRBs 051221A, 061210 , and $070724 \mathrm{~A}$, all of which have oxygen abundances of $12+\log (\mathrm{O} / \mathrm{H})=8.8-8.9$ (also using KD02; Berger 2009), although Kocevski et al. (2010) report a KD02 value of 12 $+\log (\mathrm{O} / \mathrm{H})=9.1$ for GRB 070724A. Using the same KD02 scale, our measured value for GRB $100206 \mathrm{~A}(12+\log (\mathrm{O} / \mathrm{H})=$ $\left.9.17_{-0.11}^{+0.09}\right)$ is higher than any known GRB host.

\subsection{Spatially Resolved Analysis}

The decomposition of G1 into three semi-distinct clumps (Figure 8), and the fact that the XRT error circle is nominally consistent only with the southernmost of these (although the nuclear region is only slightly outside the $90 \%$ confidence region), also motivated us to consider analysis the properties of this galaxy in a spatially resolved sense, as has been done with a handful of previous short and long GRBs (e.g., Christensen et al. 2008; Thöne et al. 2008; Rowlinson et al. 2010; Levesque \& Kewley 2007; Levesque et al. 2011).

\footnotetext{
9 Among the hosts discussed in this paragraph, only GRB 020819 was also measured on the PP04 scale: the reported value from Levesque et al. (2010c) is $8.7 \pm 0.1$, similar to what we derive for GRB $100206 \mathrm{~A}$ on the same scale.
}

We divided the two-dimensional spectrum into three spatial regions, each of $0.81(4.37 \mathrm{kpc})$ diameter and a spacing of $0{ }^{\prime} .95(5.1 \mathrm{kpc})$ between the aperture centers, and extracted the three individually as "north," "center," and "south" components. Given the uncertain slit losses and our slightly off-axis orientation, it is difficult to absolutely calibrate any of the three spectra with precision: the three spectra were summed and calibrated absolutely against the broadband photometry as before, then each spectrum's contribution to the total flux was determined by their relative synthetic photometry.

Significant differences in relative line strength and intensity (Table 4) are observed between the three regions. Most notably, nebular line fluxes in the southern part of the galaxy (nearest the XRT centroid) are significantly lower than elsewhere, both in an absolute sense $\left(F_{\lambda 6563}=16 \pm 1 \mathrm{erg} \mathrm{cm}^{-2} \mathrm{~s}^{-1}\right.$ in the southern aperture versus $56 \pm 1$ and $32 \pm 1$ in the center and north, respectively) as well as in equivalent width $\left(\mathrm{EW}_{\lambda 6563}=25.2 \pm 2.1 \AA\right.$ in the south versus $52.2 \pm 2.0 \AA$ and $38.1 \pm 2.5 \AA$ in the center and north, respectively). This indicates that the SFR in the south is lower and the average population age is older than elsewhere in the galaxy, although the amount of ongoing star formation is still quite significant (about $15 \%$ of the entire galaxy or 2-5 $M_{\odot} \mathrm{yr}^{-1}$, depending on the extinction.) The metallicity is not significantly different from that of the galaxy overall; using the same procedures employed in Section 3.4, we measure metallicity values $(12+\log (\mathrm{O} / \mathrm{H}))$ of $9.18_{-0.11}^{+0.10}$ (KD02), 8.98 -0.01 (M91), or 8.77 -0.03 (PP04), with $\log _{10}(q)=7.36_{-0.06}^{+0.62}$, consistent with the galaxy-integrated values in all cases.

\subsection{Spectral Energy Distribution}

We have recently developed a simple, flexible code for fitting multi-wavelength photometric observations of galaxies. Implemented in IDL using mpfit, the code uses a small number of smoothly varying fundamental parameters (metallicity, mass, current SFR, and parameterizations of the past star formation history) and a grid of population-synthesis models from Bruzual \& Charlot (2003) to calculate the stellar spectral energy distribution (SED). This is extinguished by dust using one of several standard extinction laws, and the absorbed energy is reradiated in the form of a multi-temperature dust graybody and midIR polycyclic aromatic hydrocarbon (PAH) emission features. Emission lines are also included, scaled using the relations from 
Table 4

Spatially Resolved Fluxes of Detected Emission Lines in G1

\begin{tabular}{|c|c|c|c|c|}
\hline Component & Line Species & $\begin{array}{c}\text { Rest Wavelength } \\
(\AA)\end{array}$ & $\begin{array}{l}\text { Unsubtracted Flux } \\
\left(10^{-17} \mathrm{erg} \mathrm{s}^{-1}\right)\end{array}$ & $\begin{array}{c}\text { Subtracted Flux } \\
\left(10^{-17} \mathrm{erg} \mathrm{s}^{-1}\right)\end{array}$ \\
\hline \multirow[t]{8}{*}{ G1-North } & {$[\mathrm{O} \mathrm{II}]^{\mathrm{c}}$} & 3727 & $8.11 \pm 1.55$ & $7.91 \pm 1.55$ \\
\hline & $\mathrm{H} \beta$ & 4861.33 & $8.11 \pm 1.24$ & $9.59 \pm 1.35$ \\
\hline & [O III] & 5006.84 & $1.01 \pm 0.98$ & $0.92 \pm 0.56$ \\
\hline & $\mathrm{H} \alpha$ & 6562.82 & $31.94 \pm 1.02$ & $33.41 \pm 1.08$ \\
\hline & {$[\mathrm{N} \mathrm{II}]$} & 6548.06 & $3.68 \pm 0.68$ & $3.84 \pm 0.72$ \\
\hline & {$[\mathrm{N}$ II $]$} & 6583.57 & $12.99 \pm 0.78$ & $13.14 \pm 0.82$ \\
\hline & {$[\mathrm{S}$ II $]$} & 6716.44 & $4.82 \pm 1.87$ & $4.44 \pm 1.73$ \\
\hline & {$[\mathrm{S} \mathrm{II}]$} & 6730.82 & $4.09 \pm 1.80$ & $4.00 \pm 1.61$ \\
\hline \multirow[t]{8}{*}{ G1-Center } & {$[\mathrm{O} \mathrm{II}]^{\mathrm{c}}$} & 3727 & $3.79 \pm 0.90$ & $5.69 \pm 1.40$ \\
\hline & $\mathrm{H} \beta$ & 4861.33 & $5.82 \pm 1.20$ & $7.57 \pm 1.24$ \\
\hline & [O III] & 5006.84 & $2.81 \pm 1.13$ & $2.63 \pm 0.96$ \\
\hline & $\mathrm{H} \alpha$ & 6562.82 & $56.43 \pm 1.34$ & $60.55 \pm 1.32$ \\
\hline & {$[\mathrm{N} \mathrm{II}]$} & 6548.06 & $8.45 \pm 0.96$ & $9.03 \pm 0.95$ \\
\hline & {$[\mathrm{N}$ II $]$} & 6583.57 & $24.27 \pm 1.08$ & $24.87 \pm 1.07$ \\
\hline & {$[\mathrm{S}$ II $]$} & 6716.44 & $10.58 \pm 2.07$ & $3.33 \pm 2.50$ \\
\hline & {$[\mathrm{S} \mathrm{II}]$} & 6730.82 & $5.80 \pm 1.81$ & $3.24 \pm 2.19$ \\
\hline \multirow[t]{8}{*}{ G1-South } & {$[\mathrm{O} \mathrm{II}]^{\mathrm{c}}$} & 3727 & $4.27 \pm 1.27$ & $4.02 \pm 1.23$ \\
\hline & $\mathrm{H} \beta$ & 4861.33 & $2.64 \pm 0.89$ & $3.61 \pm 1.02$ \\
\hline & [O III] & 5006.84 & $1.78 \pm 0.62$ & $1.26 \pm 0.53$ \\
\hline & $\mathrm{H} \alpha$ & 6562.82 & $16.17 \pm 0.78$ & $17.70 \pm 0.83$ \\
\hline & {$[\mathrm{N}$ II $]$} & 6548.06 & $1.79 \pm 0.56$ & $2.19 \pm 0.60$ \\
\hline & {$\left[\mathrm{N}_{\mathrm{II}}\right]$} & 6583.57 & $7.93 \pm 0.69$ & $8.35 \pm 0.73$ \\
\hline & {$[\mathrm{S}$ II $]$} & 6716.44 & $2.88 \pm 1.08$ & $3.06 \pm 1.09$ \\
\hline & {$[\mathrm{S}$ II $]$} & 6730.82 & $4.93 \pm 1.26$ & $4.94 \pm 1.27$ \\
\hline
\end{tabular}

Notes.

a Assuming a flat continuum, corrected for Galactic extinction.

${ }^{b}$ After subtracting a model continuum scaled to the flux of the component, corrected for Galactic extinction.

${ }^{c}$ Combined flux of both lines of the $[\mathrm{O}$ II] doublet.

Kennicutt (1998), Kewley \& Dopita (2002), and Kewley et al. (2004) using the current SFR, metallicity, and ionization.

We initially fit our models using only the broadband photometry, fixing the SFR and extinction using the $\mathrm{H} \alpha$ and $\mathrm{H} \beta$ emission-line flux measurements. However, the probability of strong, unresolved stellar absorption underlying $\mathrm{H} \beta$ makes it difficult to apply these constraints in a self-consistent way-and, furthermore, the age/extinction degeneracy makes it nearly impossible to place useful constraints on the stellar population age without photometry in the vicinity of the Balmer and $4000 \AA$ breaks. Fortunately, our flux-calibrated spectrum is of sufficient quality to fill these gaps. Using this spectrum, we calculate synthetic photometry using a series of customized "filters" covering several independent, critical regions of the spectrum-the major absorption and emission lines ([O II $],[\mathrm{O} \mathrm{III}] \lambda 5007,[\mathrm{~N} \mathrm{II}]$ $\lambda 6854,[\mathrm{~S}$ II] $\lambda \lambda 6717,6731, \mathrm{H} \delta, \mathrm{H} \gamma, \mathrm{H} \beta$, and $\mathrm{H} \alpha$ ) as well as interline continuum regions just blueward of the Balmer break (5650-5730 $\AA$ and 5850-6050 $\AA$ ). (With the exception of the [O II] line, we do not attempt this technique blueward of the Balmer break due to the weak continuum trace and relatively large uncertainty in the overall flux calibration of the blue side.) We exclude the W3 filter in these fits, since the model is subject to large systematic uncertainties in this wavelength region regarding the fraction of dust emission in PAH lines and the possible presence of highly embedded star formation. (However, all of our models which reasonably fit the optical/NIR points also accurately predicted the W3 flux within $2 \sigma$, and including this filter does not qualitatively change any results.)

Three different models of the past star formation history were attempted (see inset of Figure 9): purely constant (up to and including the present-day value), constant (with an instantaneous change at $t=10^{7} \mathrm{yr}$ ), and exponentially falling with a 100 Myr $e$-folding time (also with a step at $t=10^{7} \mathrm{yr}$ ). For each of these star formation histories, we attempted two models of the dust attenuation: either a single dust screen applied to all stars uniformly, or with two different screens-one applied to the young $(<100 \mathrm{Myr})$ stars and nebular lines, the other to older stars ( $>100$ Myr). We try Small Magellanic Cloud (Gordon et al. 2003), Milky Way (Cardelli et al. 1989), and Calzetti (Calzetti et al. 2000) extinction "laws" for each fit (Local-Group-type extinction as implemented using the generalized parameterization of Fitzpatrick \& Massa 1990) and report the result producing the best $\chi^{2}$ in Table 5 .

With the exception of the purely constant, single-extinction model (which produces a poor fit), all of these models produce reasonable fits to the combined broadband and pseudonarrowband photometry of G1. These different models are shown in Figure 9, with the parameters outlined in Table 5. Different assumptions naturally lead to an intrinsic dispersion of properties, but essentially all good fits share several features in common. The current SFR is large but not extreme (20-40 $\left.M_{\odot}\right)$, and the stellar mass is also quite high, $(3-7) \times 10^{10} M_{\odot}$. The (maximum) age of the stellar population is at least $0.5 \mathrm{Gyr}$ (at least $1 \mathrm{Gyr}$ for the constant star formation history). The extinction is also large, $A_{V}=1.8 \pm 0.2 \mathrm{mag}$ (for a single extinction) or $A_{V, \text { young }}=2.7 \pm 0.4 \mathrm{mag}$ and $A_{V \text {,old }}=1.1 \pm 0.2 \mathrm{mag}$ (age-dependent extinction). All models require a large bolometric luminosity of $L=(3-4) \times 10^{11} L_{\odot}$, the large majority of which is emitted in the far-IR, thus classifying the galaxy as an unambiguous LIRG. As we do not have any far-IR 


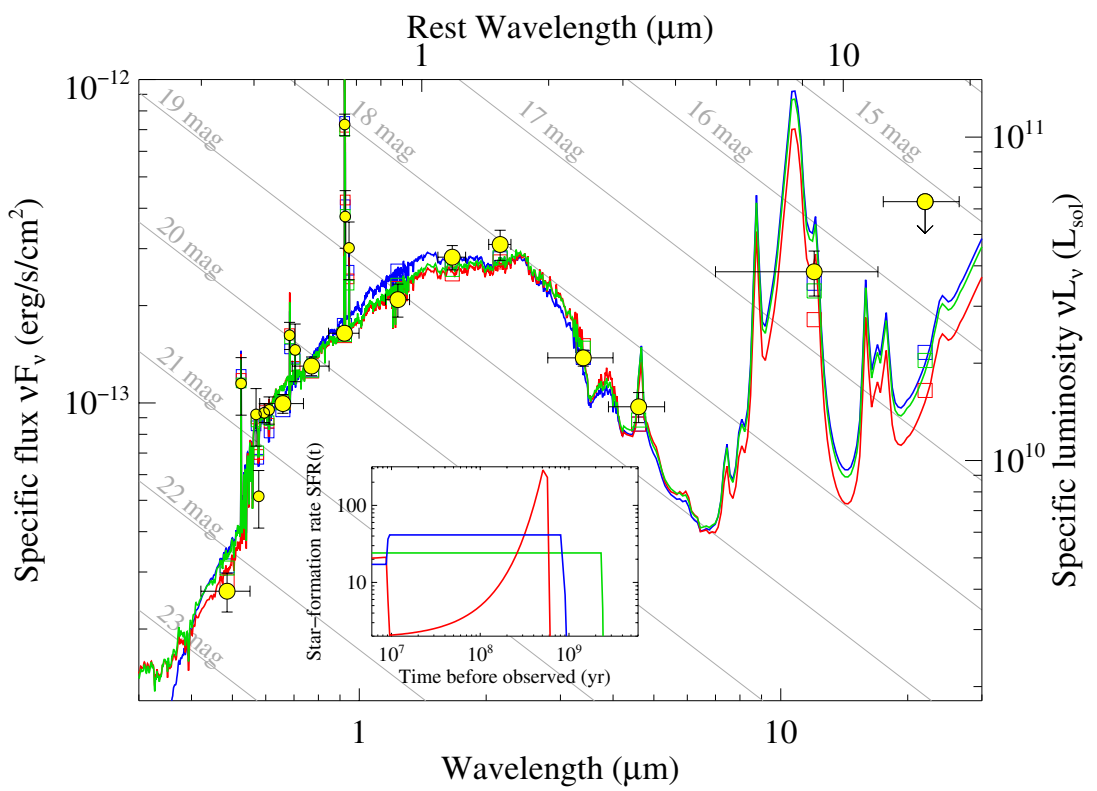

Figure 9. Photometry of the putative host galaxy G1 of GRB 100206A, fit with stellar population models using our own implementation of the Bruzual \& Charlot (2003) templates (including nebular lines, dust extinction, and mid/far-IR dust emission) for different assumptions of the star formation history. The broadband photometry is supplemented by synthetic narrowband photometry of major emission and absorption-line regions (and interline regions near the Balmer break). Three different star formation history models are shown, all of which produce similar results (see also Table 5). The green curve shows a strictly continuous star formation history from the formation of the galaxy until the present time. The blue curve is also constant, except for an instantaneous change in the recent past. The red curve assumes an impulsive star formation episode at some point in the past with exponential decay time $\tau=200 \mathrm{Myr}$. All three models use an age-dependent dust screen. Broadband photometry is indicated with large yellow points; synthetic narrowband photometry is indicated with smaller points. Empty colored squares show the synthetic fluxes for each filter. The gray lines show contours of constant AB magnitude.

(A color version of this figure is available in the online journal.)

Table 5

Results of Model Fits to Photometry and Spectroscopy of G1

\begin{tabular}{|c|c|c|c|c|c|c|c|c|c|}
\hline$\overline{Z / Z_{\odot}{ }^{\mathrm{a}}}$ & $\begin{array}{c}\mathrm{SFR}_{0}{ }^{\mathrm{b}} \\
\left(M_{\odot} \mathrm{yr}^{-1}\right)\end{array}$ & $\begin{array}{c}\mathrm{SFR}_{\mathrm{av}}{ }^{\mathrm{c}} \\
\left(M_{\odot} \mathrm{yr}^{-1}\right)\end{array}$ & $\begin{array}{l}\text { Age }^{\mathrm{d}} \\
(\mathrm{Gyr})\end{array}$ & $\begin{array}{c}\tau^{\mathrm{e}} \\
(\mathrm{Myr})\end{array}$ & $\begin{array}{c}\operatorname{Mass}^{\mathrm{f}} \\
\left(10^{9} M_{\odot}\right)\end{array}$ & $\begin{array}{l}\text { Dust } \\
\text { Law }^{g}\end{array}$ & $\begin{array}{c}A_{V}(\mathrm{old})^{\mathrm{h}} \\
(\mathrm{mag})\end{array}$ & $\begin{array}{c}A_{V} \text { (young) } \\
\quad(\text { mag })\end{array}$ & $\chi^{2}$ \\
\hline $2.56 \pm 0.51$ & \multicolumn{2}{|c|}{$11.6 \pm 1.8$} & $11.5 \pm 3.3$ & $\infty$ & $133 \pm 31$ & $\mathrm{smc}$ & \multicolumn{2}{|c|}{$1.2 \pm 0.1$} & $25.47 / 14$ \\
\hline $2.16 \pm 0.46$ & $17.0 \pm 3.0$ & $41.0 \pm 39.4$ & $0.9 \pm 0.8$ & $\infty$ & $36 \pm 14$ & $\mathrm{smc}$ & \multicolumn{2}{|c|}{$1.8 \pm 0.2$} & $17.00 / 13$ \\
\hline $2.58 \pm 0.36$ & $19.4 \pm 4.2$ & $94.3 \pm 26.6$ & $0.6 \pm 0.2$ & 100 & $54 \pm 5$ & calz & \multicolumn{2}{|c|}{$1.8 \pm 0.2$} & $13.48 / 13$ \\
\hline $2.32 \pm 0.36$ & \multicolumn{2}{|c|}{$24.0 \pm 6.3$} & $2.4 \pm 0.9$ & $\infty$ & $57 \pm 16$ & calz & $1.3 \pm 0.1$ & $2.2 \pm 0.3$ & $12.82 / 13$ \\
\hline $1.94 \pm 0.75$ & $37.4 \pm 13.1$ & $18.9 \pm 14.0$ & $3.4 \pm 2.3$ & $\infty$ & $65 \pm 21$ & calz & $1.1 \pm 0.2$ & $2.7 \pm 0.4$ & $9.16 / 12$ \\
\hline $2.27 \pm 0.46$ & $34.1 \pm 14.6$ & $82.0 \pm 24.4$ & $0.6 \pm 0.2$ & 100 & $52 \pm 5$ & calz & $1.6 \pm 0.3$ & $2.5 \pm 0.5$ & $11.88 / 12$ \\
\hline
\end{tabular}

Notes.

${ }^{\mathrm{a}}$ Gas-phase oxygen abundance (in solar units, for $\log [\mathrm{O} / \mathrm{H}]_{\odot}=8.69$ ).

${ }^{\mathrm{b}}$ Current SFR.

${ }^{c}$ Average SFR.

${ }^{\mathrm{d}}$ Age of formation.

${ }^{\mathrm{e}} \mathrm{SFR}$ decay timescale (for exponential-decline model).

${ }^{\mathrm{f}}$ Total stellar mass.

${ }^{\mathrm{g}}$ Best-fit dust extinction curve (Milky Way, Small Magellanic Cloud, or Calzetti).

${ }^{\mathrm{h}}$ Extinction for $t>100 \mathrm{Myr}$ stars.

${ }^{\mathrm{i}}$ Extinction for $t<100 \mathrm{Myr}$ stars.

measurements directly sampling the thermal dust emission, it is possible that the luminosity is even higher than this if an additional deeply embedded $\left(A_{V}>50 \mathrm{mag}\right)$ component is present in the galaxy. However, the relatively modest mid-IR emission in the W3 filter and the W4 nondetection suggest that such an extra component is not likely to be present, as is the case for most high-redshift LIRGs (Reddy et al. 2010), but unlike nearby LIRGs and ULIRGs (e.g., Symeonidis et al. 2008).

G2 is only detected in four filters, and no hydrogen lines are evident in the spectrum, so it is not possible to constrain the properties of the galaxy in any detail. We modeled the spectrum and SED using the same procedure as for G1 above using our broadband photometry and emission-line synthetic photometry. Assuming no extinction and a constant star formation history, the galaxy is consistent with being a low-mass, non-starbursting galaxy with a modest current SFR of $\sim 0.5 M_{\odot} \mathrm{yr}^{-1}$ and mass $\sim 4 \times 10^{9} M_{\odot}$.

\section{DISCUSSION}

\subsection{Association of G1 with GRB 100206 A}

We have previously suggested that the probability of chance alignment of an IR-bright source with an XRT position is very low. Here, we quantify this calculation, and demonstrate that the 
Table 6

Chance Alignment Parameters for G1 and G2

\begin{tabular}{lccccc}
\hline \hline Galaxy & Filter & $\begin{array}{c}m^{\mathrm{a}} \\
(\mathrm{mag})\end{array}$ & $\begin{array}{c}n_{m<m_{\mathrm{G} 1}}^{\mathrm{b}} \\
\left(\mathrm{deg}^{-1} \mathrm{mag}^{-1}\right)\end{array}$ & $P_{\text {chance }}^{\mathrm{c}}$ & $P_{\text {chance, allSHBs }}^{\mathrm{d}}$ \\
\hline $\mathrm{G} 1$ & $g$ & 22.33 & 5730 & 0.05 & 0.80 \\
& $R$ & 20.38 & 3160 & 0.03 & 0.58 \\
& $i$ & 20.09 & 2280 & 0.02 & 0.46 \\
& $z$ & 19.67 & 2280 & 0.02 & 0.47 \\
& $J$ & 18.17 & 2000 & 0.02 & 0.42 \\
& $H$ & 17.04 & 820 & 0.007 & 0.20 \\
& $K_{s}$ & 16.19 & 740 & 0.006 & 0.18 \\
& $3.6 \mu \mathrm{m}$ & 15.74 & 860 & 0.008 & 0.21 \\
& $4.5 \mu \mathrm{m}$ & 15.14 & 950 & 0.008 & 0.23 \\
$\mathrm{G} 2$ & 11.23 & 170 & 0.0015 & 0.04 \\
\hline & & 25.21 & $1.1 \times 10^{5}$ & 0.61 & 1.0 \\
& & 24.19 & $7.5 \times 10^{4}$ & 0.48 & 1.0 \\
& & 23.95 & $8.3 \times 10^{4}$ & 0.52 & 1.0 \\
& & 23.62 & $1.0 \times 10^{5}$ & 0.58 & 1.0 \\
\hline
\end{tabular}

Notes.

a Apparent magnitude, corrected for Galactic extinction.

b Density of galaxies on the sky brighter than $m$.

c Probability of a 3."3 XRT error circle overlapping an equivalent or brighter galaxy by chance.

d Probability of at least one error circle among all X-ray-detected Swift short bursts from 2005 to 2011 overlapping an equivalent or brighter galaxy by chance.

case for associating GRB 100206A with the galaxy G1 is quite strong.

Figure 1 shows the current XRT error circle relative to our optical imaging. Note that while the galaxy is centered outside the XRT error circle, a significant amount of flux from the southern end of the disk is in fact contained within the XRT error circle.

Roughly following Bloom et al. (2002), an estimate of the probability of chance association $P_{\text {chance }}$ can be provided by

$$
P_{\text {chance }}=1-\exp \left(-A_{\text {assoc }} \times n_{m<m_{\text {obj }}}\right) \text {. }
$$

Here, $A_{\text {assoc }}$ is the area on the sky of the region in which an error circle centered in that region would still lead us to connect the GRB and the galaxy. We take this as a circle of radius equal to the sum of the galaxy's major axis and the $90 \%$ confidence radius of the XRT position: $\sim 6^{\prime \prime}$, so $A_{\text {assoc }}=113 \operatorname{arcsec}^{2}$.

The term $n_{m<m_{\mathrm{obj}}}$ is the density on the sky of objects of equal or greater brightness. Because of G1's unusual colors, this factor is strongly dependent on the filter chosen. Rather than restricting our calculation to a single filter, then, we calculate $P_{\text {chance }}$ for all filters in which the galaxy was detected. The number density of galaxies on the sky for various filters was taken from a variety of sources: Yasuda et al. (2001) for the Sloan Digital Sky Survey giz bands, Hogg et al. (1997) for the $R$ band, and Jarrett (2004), Frith et al. (2006), Imai et al. (2007), and Maihara et al. (2001) for the $J, H$, and $K$ bands. For the W3 filter, we use the number-count plots in Jarrett et al. (2011), while for the W1 and W2 filters we adopt the more precisely determined Spitzer/IRAC number counts in Fazio et al. (2004), transforming the WISE filters to equivalent IRAC fluxes using synthetic photometry of our model. Results were checked for consistency with the compilation graphs at http://astro.dur.ac.uk/ nm/pubhtml/counts/counts.html for relevant filters.

The sky densities and $P_{\text {chance }}$ values are presented in Table 6 . The fourth column lists the basic $P_{\text {chance }}$ as described above, the value of which is small almost regardless of the filter examined-ranging from a few percent in the optical filters down to $\sim 10^{-3}$ in the W3 filter, similar to the strength of association of GRB 050509B to its putative elliptical host (Bloom et al. 2006, which was based on $r$-band photometry) and indeed stronger than many long-GRB host associations (e.g., Bloom et al. 2002).

To adopt a more skeptical perspective, it should be noted that the number of localized short GRBs is no longer small, and curious chance alignments are bound to happen eventually. We therefore also calculate the $P_{\text {chance }}$ using the combined area covered by all 46 short GRBs with X-ray localizations through the end of 2011: $733 \operatorname{arcsec}^{2}$ or (adding the $3^{\prime \prime}$ major axis of the galaxy) $3600 \operatorname{arcsec}^{2}$. The value of $P_{\text {chance }}$ is between 0.18 and 0.8 depending on the filter, an obviously less convincing result, indicating that the global short-GRB sample size is indeed approaching a point where bright foreground or background interloper galaxies can be expected somewhere in the full sample. However, when comparing to the W3 filter magnitude-where the unusual characteristics of this galaxy are most pronounced - the probability is still quite low at only 0.04 . In other words, the current short-GRB sample size is still a factor of 5-10 too small for chance alignment with a comparably mid-IR bright galaxy to be expected.

An alternative hypothesis that GRB 100206A originates from G2 was first presented by Berger et al. (2010). While this certainly cannot be ruled out, we judge it to be relatively unlikely given the faintness of this galaxy and the size of the XRT error circle: for all four filters, $P_{\text {chance }}$ of the individual association is $\sim 0.5$ (i.e., a randomly positioned error circle of this size will enclose a comparable source approximately half the time). Also, G2 (like G1) is actually centered outside the $90 \%$ error circle, but the galaxy's small radius (unlike G1, G2 is unresolved in 0 .7 seeing) places very little flux significantly inside the $90 \%$ confidence region.

Based on these considerations, we argue that the host galaxy of this event is G1. As with all short-GRB host associations to date, this one is based entirely on a posteriori arguments and-given the absence of an absorption spectrum-is impossible to prove conclusively. If this GRB is associated, however, then the unique properties of this galaxy (among short-GRB hosts so far) have the potential to impact our understanding of the short-GRB progenitor in important ways. In the remainder of the paper, we will assume that this association is correct and examine its implications.

\subsection{Characteristics of the Galaxy and Implications for the Progenitor}

With an SFR of at least $15 M_{\odot} \mathrm{yr}^{-1}$ (and 30-40 $M_{\odot} \mathrm{yr}^{-1}$ in our best-fit models), G1 is the most rapidly star-forming host of a short-hard GRB to date. The next-highest reported SFR among short-GRB hosts in the literature is only $6.1 M_{\odot} \mathrm{yr}^{-1}$ (GRB 060801; Berger 2009), much less than our inferred value. (After that, the next-highest short-GRB SFRs belong to GRBs 061217 and 070724 , both at a mere $2.5 M_{\odot} \mathrm{yr}^{-1}$.) ${ }^{10}$ The intense ongoing star formation raises the possibility that the GRB itself may be

\footnotetext{
10 While extreme, the qualitative properties of G1 are not completely without precedent among short-GRB hosts. The host of GRB 070724 (Berger et al. 2009; Kocevski et al. 2010) in particular, despite having an SFR and stellar mass an order of magnitude lower than those of G1, is also well above the average among short-GRB hosts in both these properties. The host of GRB 070724 is also significantly dust-extinguished, and in fact has a dust-reddened optical afterglow.
} 
the product of a short-lived star, coming from a young stellar progenitor produced at the same time as the large population of massive stars currently powering the bright nebular and midIR emission. However, more detailed analysis of the galaxy suggests that a large population of evolved stars must be present as well, and that the current SFR is not even unusual relative to the rest of the galaxy's history.

Unfortunately, there is no direct means of determining which of these two populations produced the GRB. While the XRT position seems to favor an origin within the (relatively) less actively star-forming southern part of the galaxy, neither the accuracy of this position nor the resolution of our imaging of the host is sufficient to actually determine whether the burst originated within a region of active star formation or not.

Bereft of any direct means of evaluating the age of the progenitor-and, thus far, only a single example of a dusty, rapidly star forming, short-GRB host to date-we must resort again to statistical arguments. Relative to the universe as a whole, is this galaxy more likely to produce explosions from old progenitors, or young ones? To phrase this question more precisely (under the simplifying assumption that the shortGRB rate is directly proportional to the number of stars of the appropriate age range and ignoring secondary effects such as detection biases), we can ask the following: as a relative fraction of all stars in the universe at that redshift, are there more "old" stars or "young" stars in LIRG-like hosts at $z \approx 0.4$ ? (For now, we will encapsulate the "unusual" characteristics of this host galaxy entirely in its designation as an LIRG.) Fortunately, this type of question has been addressed in great detail by recent studies: the sites of star formation, the build-up of stellar mass, and the role of the LIRG phase in galaxy evolution have been major foci of recent work in extragalactic astronomy.

We will first address the fraction of star formation occurring in LIRGs. At higher redshifts of $z>1$, a quite significant fraction of all star formation occurs in luminous, dusty galaxies (as much as 70\%; Le Floc'h et al. 2005). However, this fraction begins to fall rapidly below $z=1$ and it is already relatively low by $z=0.4$ : Figure 14 of Le Floc'h et al. (2005) indicates that about $20 \%$ of star formation at that era occurs in LIRGs, and a consistent value of $10 \%-20 \%$ is given by Pérez-González et al. (2005). It is therefore not unexpected that if a small fraction of short GRBs were produced by young ( $<100 \mathrm{Myr}$ ) stars, we might have observed one in an LIRG-though it is likely that several other short GRBs would be observed to occur in more "typical" star-forming galaxies first. (Whether this has in fact occurred is a matter of debate: see Section 1.)

The fraction of all stellar mass in LIRGs at moderate redshift is given by Caputi et al. (2006), who estimate that $24 \%$ of the stellar mass at $z=0.5-1.0$ is in LIRGs and ULIRGs. The fraction at lower redshifts is not given, but Figure 8 of Salim et al. (2009) suggests it is similar (within a factor of a few) to that at $z \approx 0.5-1.0$; the number of LIRGs does not begin to fall precipitously to the local density until about $z \lesssim 0.3$, when they essentially disappear in deep surveys. The fraction of all stars cosmologically that are present in LIRGs in this redshift range is therefore about $10 \%-20 \%$, quite comparable to the cosmological fraction of young stars in LIRGs. Even by a posteriori arguments, then, we are unable to associate this burst with a specific stellar population age with any confidence.

We can also examine the galaxy's morphological properties for clues about the GRB's origin. At $z \approx 0$, for example, LIRGs are predominantly or entirely the products of major mergers: transient and (relatively) rare phenomena in the life of a galaxy with clear observational signatures, such as nuclear starbursts and tidal features. (This is not the case at $z \approx 1$, when merging systems become a minority among LIRGs/ULIRGs; Zheng et al. 2004.) Independent of the SED, then, a GRB from within a merger system could be seen as an indication of association with a progenitor with age similar to the merger itself, on the grounds that the explosion of an older star by chance during the short-lived merger phase would be an unlikely coincidence. No such signatures are present; while our resolution is not sufficient to robustly rule out a merger remnant, the majority of the galaxy's flux appears to be associated with an ordinary exponential disk. The galaxy is slightly asymmetric, in that the region of maximum flux is displaced by $\sim 1 \mathrm{kpc}$ from the center of the outer isophotes, but this does not appear to reflect the detailed properties of the galaxy. Similarly, there are no obvious disturbances in the structure of the rotation curve (Figure 4).

Additionally, we can examine the physical characteristics of the galaxy from our SED modeling. Evidence for recent, significant elevation in the SFR above its historical average (equivalently, a large specific star formation rate, SFR/ $\left.M_{*} \gg 1 / t\right)$ would also be indicative that the GRB caught this galaxy in an unusual, transient part of its history, which is much more likely for a young progenitor than an older one. But again, there is no such evidence: the current and past SFRs are comparable, and the sSFR is relatively unremarkable $\left(\mathrm{SFR} / M_{*} \approx 0.5 \mathrm{Gyr}^{-1}\right)$. This is the largest value for a shortGRB host yet $\left(\mathrm{SFR} / M_{*}\right.$ ranges between 0 and $0.2 \mathrm{Gyr}^{-1}$ using the SFRs from Berger 2009 and masses from Leibler \& Berger 2010 ), but only by a factor of two to three. The value does overlap with the distribution of (massive-star associated) long-GRB hosts (typical values of SFR/ $M_{*}$ range from 0.1 to $50 \mathrm{Gyr}^{-1}$; Savaglio et al. 2009; Castro Cerón et al. 2010), but only at the low end: it is not an extreme value indicating an unambiguously starbursting galaxy.

Finally, we can examine the implication of the resolved spectral analysis of the host. In general, the southern part of the galaxy (that contained within the $90 \%$ confidence error circle) has a lower SFR than the rest of the object, representing only about $15 \%$ of the total contribution of the whole disk. If the GRB were produced by a massive star (or other shortlived object) it would have to explode at its birth site, and an origin in a more rapidly star-forming part of the galaxy might be expected. If the GRB originated from an older progenitor (and in particular a "kicked" object such as a neutron star binary), then it could explode anywhere within the galaxy (or its halo) and the observed location is somewhat more natural. On the other hand, the SFR in the southern disk is still significant, and even an origin near the rapidly star-forming center is not completely ruled out by the XRT position, so this argument is not conclusive.

All available evidence, therefore, suggests that this is a relatively ordinary $z \approx 0.4$ galaxy when weighted either by star formation or by stellar mass. Furthermore, it is (probably) being observed at a fairly typical moment in its history, with no clear indication of a large, recent increase in the SFR related to a merger or other transient event. The properties of the galaxy give no clear means of distinguishing a short-lived from a long-lived progenitor of this system.

\section{CONCLUSIONS}

Seen as an isolated event, the age of the progenitor of GRB 100206A is frustratingly inconclusive: essentially every diagnostic we have applied to the host system is consistent 
with both a short-lived progenitor $(<10 \mathrm{Myr})$ and a longlived one ( $>1 \mathrm{Gyr})$. While the galaxy has a large current SFR $\left(\sim 30 M_{\odot} \mathrm{yr}^{-1}\right)$, this is matched by a substantial pre-existing stellar mass $\left(>10^{11} M_{\odot}\right)$. This, and a largely ordinary disk-like morphology, suggest a steady star formation history and give no direct indication of the progenitor's age. The lower SFR in the southern part of the galaxy closest to the XRT centroid points toward an older origin, but given the size of the error circle and existence of significant star formation throughout the host at the resolution of our observations this is far from conclusive.

Seen in the broader context of other short-GRB host-galaxy work, however, our results-even those which were individually inconclusive - can be seen as generally supportive of the prevailing paradigm. As discussed in Section 4.2, the significant amount of stellar mass present in LIRG systems at $z>0.3$ suggests that it should be no surprise that a few percent of short GRBs should occur within these massive and actively starforming galaxies, even if their progenitor is exclusively a longlived object such as a neutron-star binary. The fraction should be even higher if some of the detection biases are considered: preferential ejection of the progenitor system from low-mass host galaxies (due to supernova "kicks"), as well as preferential detection of X-ray or optical afterglow from systems in dense environments, would both have much less impact on massive galaxies such as G1 than on more typical lower-mass galaxies (which would be actively selected against), favoring the discovery of this type of host. Given what has become a quite large short-GRB sample (46 objects with X-ray or optical localizations and at least 20 likely host galaxies), the occurrence of an event in an LIRG is not only unsurprising for an older progenitor, but could have been predicted.

This, coupled to the lack of any definitive signature tying GRB 100206A to recent star formation in its host galaxy despite careful scrutiny of several possible lines of argument (including the SED-inferred star formation history and morphology), can be interpreted as evidence that this event is the product of a relatively mature progenitor system in the galaxy. Given the precedent set by other short GRBs, the most natural explanation for this burst is that it was produced in the same manner as the rest of the population-for which a compelling statistical case is now building for a generally old ( $>100 \mathrm{Myr}$ ) progenitor, even if the delay-time distribution is indeed broad.

Of course, a younger progenitor is not ruled out. If this event were the result of a young progenitor (and especially a massive star), then it is interesting to note that the high metallicity of this system — at least solar and perhaps significantly higher-would nevertheless be indicative of an origin distinct from that of long GRBs, which quite notably avoid dusty, massive LIRG-like systems in the local universe. In that case, this event would be more indicative of a broad delay-time distribution for the shortGRB population, rather than an origin from the same population that produces LGRBs due to, for example, off-axis collapsars (Lazzati et al. 2010). While it is possible that a few long-duration GRBs may occur in high-metallicity regions (Levesque et al. 2010c), these appear to be relatively rare (Stanek et al. 2006; Modjaz et al. 2008; Savaglio et al. 2009; Levesque et al. 2010b), so even if this event were to be associated with the youngest stellar population in this galaxy, it would nevertheless continue to support a distinction between the progenitor systems of short and long GRBs.

Support for this work was provided by NASA through Hubble Fellowship grant HST-HF-51296.01-A awarded by the Space
Telescope Science Institute, which is operated by the Association of Universities for Research in Astronomy, Inc., for NASA, under contract NAS 5-26555. A.V.F. and S.B.C. acknowledge generous support from Gary and Cynthia Bengier, the Richard and Rhoda Goldman Fund, NASA/Swift grants NNX10AI21G and NNX12AD73G, and NSF grant AST-0908886.

PAIRITEL is operated by the Smithsonian Astrophysical Observatory (SAO) and was made possible by a grant from the Harvard University Milton Fund, a camera loan from the University of Virginia, and continued support of the SAO and UC Berkeley. The PAIRITEL project is further supported by NASA/Swift Guest Investigator grant NNX08AN84G and NNX10AI28G. We acknowledge support from the NSF/AAG 1009991.

We wish to acknowledge N. R. Tanvir, A. J. Levan, E. Berger, and R. Chornock, as well as the Gemini-North staff (in particular T. Geballe, M. Lemoine-Busserolle, S. Cote, and R. Mason) for acquisition of the Gemini data. We also heartily thank J. M. Silverman for acquisition of the second epoch of Keck spectroscopy. The W. M. Keck Observatory is operated as a scientific partnership among the California Institute of Technology, the University of California, and NASA. The Observatory was made possible by the generous financial support of the W. M. Keck Foundation. We wish to extend special thanks to those of Hawaiian ancestry on whose sacred mountain we are privileged to be guests.

This work made use of data supplied by the UK Swift Science Data Centre at the University of Leicester. It is a pleasure to thank all members of the Swift team, who built and continue to operate this successful mission. This publication makes use of data products from the Wide-field Infrared Survey Explorer, which is a joint project of the University of California, Los Angeles, and the Jet Propulsion Laboratory/California Institute of Technology, funded by the National Aeronautics and Space Administration. This research also made use of the NASA/ IPAC Extragalactic Database (NED), which is operated by the Jet Propulsion Laboratory, California Institute of Technology, under contract with NASA. The National Energy Research Scientific Computing Center, which is supported by the Office of Science of the U.S. Department of Energy under contract No. DE-AC02-05CH11231, provided staff, computational, research, and data storage in support of this project. We thank P. Nugent for providing the DeepSky imaging.

D. P. acknowledges useful discussions with Evan Kirby, Vivian U, and Enrico Ramirez-Ruiz, and helpful comments on the manuscript from D. A. Kann, as well as the helpful commentary and suggestions of the referee.

Facilities: $\quad$ Swift, Keck:I (LRIS), Gemini:Gillett (GMOS), WISE

\section{REFERENCES}

Andreev, M., Sergeev, A., Parakhin, N., et al. 2010, GCN Circ., 10455 Asplund, M., Grevesse, N., Sauval, A. J., \& Scott, P. 2009, ARA\&A, 47, 481 Baldwin, J. A., Phillips, M. M., \& Terlevich, R. 1981, PASP, 93, 5 Band, D., Matteson, J., Ford, L., et al. 1993, ApJ, 413, 281

Barthelmy, S. D., Barbier, L. M., Cummings, J. R., et al. 2005, Space Sci. Rev., 120,143

Berger, E. 2009, ApJ, 690, 231

Berger, E. 2010, ApJ, 722, 1946

Berger, E., Cenko, S. B., Fox, D. B., \& Cucchiara, A. 2009, ApJ, 704, 877

Berger, E., Chornock, R., Tanvir, N., et al. 2010, GCN Circ., 10395

Berger, E., Price, P. A., Cenko, S. B., et al. 2005, Nature, 438, 988

Bertin, E., \& Arnouts, S. 1996, A\&AS, 117, 393

Bhattacharya, D., Mohan, V., Ramaprakash, A. N., Rajarshi, C., \& Misra, K. 2010, GCN Circ., 10380 
Bloom, J. S., Butler, N. R., \& Perley, D. A. 2008, in AIP Conf. Proc. 1000, Gamma-ray Bursts, ed. M. Galassi, D. Palmer, \& E. Fenimore (Melville, NY: AIP), 11

Bloom, J. S., Kasen, D., Shen, K. J., et al. 2012, ApJ, 744, L17

Bloom, J. S., Kulkarni, S. R., \& Djorgovski, S. G. 2002, AJ, 123, 111

Bloom, J. S., \& Prochaska, J. X. 2006, in AIP Conf. Proc. 836, Gamma-ray Bursts in the Swift Era, ed. S. S. Holt, N. Gehrels, \& J. A. Nousek (Melville, NY: AIP), 473

Bloom, J. S., Prochaska, J. X., Pooley, D., et al. 2006, ApJ, 638, 354 Bresolin, F., Gieren, W., Kudritzki, R.-P., et al. 2009, ApJ, 700, 309

Bruzual, G., \& Charlot, S. 2003, MNRAS, 344, 1000

Burrows, D. N., Hill, J. E., Nousek, J. A., et al. 2005, Space Sci. Rev., 120, 165

Butler, N. R., Kocevski, D., Bloom, J. S., \& Curtis, J. L. 2007, ApJ, 671, 656

Calzetti, D., Armus, L., Bohlin, R. C., et al. 2000, ApJ, 533, 682

Caputi, K. I., Dole, H., Lagache, G., et al. 2006, A\&A, 454, 143

Cardelli, J. A., Clayton, G. C., \& Mathis, J. S. 1989, ApJ, 345, 245

Castro Cerón, J. M., Michałowski, M. J., Hjorth, J., et al. 2010, ApJ, 721, 1919

Cenko, S. B., Bloom, J. S., Perley, D. A., et al. 2010, GCN Circ., 10389

Cenko, S. B., Fox, D. B., Moon, D.-S., et al. 2006, PASP, 118, 1396

Christensen, L., Vreeswijk, P. M., Sollerman, J., et al. 2008, A\&A, 490, 45

Di Matteo, P., Bournaud, F., Martig, M., et al. 2008, A\&A, 492, 31

Eichler, D., Livio, M., Piran, T., \& Schramm, D. N. 1989, Nature, 340, 126

Fazio, G. G., Ashby, M. L. N., Barmby, P., et al. 2004, ApJS, 154, 39

Fitzpatrick, E. L., \& Massa, D. 1990, ApJS, 72, 163

Fong, W., Berger, E., Chornock, R., et al. 2011, ApJ, 730, 26

Fong, W., Berger, E., \& Fox, D. B. 2010, ApJ, 708, 9

Fox, D. B., Frail, D. A., Price, P. A., et al. 2005, Nature, 437, 845

Frith, W. J., Metcalfe, N., \& Shanks, T. 2006, MNRAS, 371, 1601

Fruchter, A. S., Levan, A. J., Strolger, L., et al. 2006, Nature, 441, 463

Fryer, C. L., Mazzali, P. A., Prochaska, J., et al. 2007, PASP, 119, 1211

Fynbo, J. P. U., Watson, D., Thöne, C. C., et al. 2006, Nature, 444, 1047

Gal-Yam, A., Fox, D. B., Price, P. A., et al. 2006, Nature, 444, 1053

Gehrels, N., Chincarini, G., Giommi, P., et al. 2004, ApJ, 611, 1005

Gehrels, N., Norris, J. P., Barthelmy, S. D., et al. 2006, Nature, 444, 1044

Gehrels, N., Sarazin, C. L., O’Brien, P. T., et al. 2005, Nature, 437, 851

Goad, M. R., Tyler, L. G., Beardmore, A. P., et al. 2007, A\&A, 476, 1401

Gordon, K. D., Clayton, G. C., Misselt, K. A., Landolt, A. U., \& Wolff, M. J. 2003, ApJ, 594, 279

Graham, J., Fruchter, A. S., Kewley, L. J., et al. 2010, BAAS, 42, 229

Graham, J., Fruchter, A. S., Levesque, E. M., et al. 2011, BAAS, 43, 217

Guziy, S., Aceituno, F. J., \& Castro-Tirado, A. J. 2010, GCN Circ., 10384

Hirschi, R., Meynet, G., \& Maeder, A. 2005, A\&A, 443, 581

Hjorth, J., Sollerman, J., Gorosabel, J., et al. 2005, ApJ, 630, L117

Hogg, D. W., Pahre, M. A., McCarthy, J. K., et al. 1997, MNRAS, 288, 404

Imai, K., Matsuhara, H., Oyabu, S., et al. 2007, AJ, 133, 2418

Jakobsson, P., Hjorth, J., Fynbo, J. P. U., et al. 2004, ApJ, 617, L21

Jarrett, T. 2004, PASA, 21, 396

Jarrett, T. H., Cohen, M., Masci, F., et al. 2011, ApJ, 735, 112

Jester, S., Schneider, D. P., Richards, G. T., et al. 2005, AJ, 130, 873

Kann, D. A., Klose, S., Zhang, B., et al. 2011, ApJ, 734, 96

Kennicutt, R. C., Jr. 1998, ARA\&A, 36, 189

Kewley, L. J., \& Dopita, M. A. 2002, ApJS, 142, 35

Kewley, L. J., \& Ellison, S. L. 2008, ApJ, 681, 1183

Kewley, L. J., Geller, M. J., \& Jansen, R. A. 2004, AJ, 127, 2002

Kienlin, A. V. 2010, GCN Circ., 10381

Kocevski, D., Thöne, C. C., Ramirez-Ruiz, E., et al. 2010, MNRAS, 404, 963

Kouveliotou, C., Meegan, C. A., Fishman, G. J., et al. 1993, ApJ, 413, L101

Krimm, H. A., Cummings, J. R., Evans, P. A., \& Marshall, F. E. 2010, GCN Rep., 271.1

Kulkarni, S. R. 2005, arXiv:astro-ph/0510256

Kuroda, D., Yanagisawa, K., Shimizu, Y., et al. 2010, GCN Circ., 10388

Landolt, A. U. 2009, AJ, 137, 4186

Lazzati, D., Morsony, B. J., \& Begelman, M. C. 2010, ApJ, 717, 239

Le Floc'h, E., Papovich, C., Dole, H., et al. 2005, ApJ, 632, 169

Leibler, C. N., \& Berger, E. 2010, ApJ, 725, 1202

Leloudas, G., Xu, D., Malesani, D., et al. 2010, GCN Circ., 10387

Levan, A. J., Tanvir, N. R., Wiersema, K., et al. 2010, GCN Circ., 10386

Levesque, E. M., Berger, E., Soderberg, A. M., \& Chornock, R. 2011, ApJ, 739,23

Levesque, E. M., Bloom, J. S., Butler, N. R., et al. 2010a, MNRAS, 401, 963

Levesque, E. M., \& Kewley, L. J. 2007, ApJ, 667, L121

Levesque, E. M., Kewley, L. J., Berger, E., \& Zahid, H. J. 2010b, AJ, 140, 1557
Levesque, E. M., Kewley, L. J., Graham, J. F., \& Fruchter, A. S. 2010c, ApJ, 712, L26

Li, L.-X., \& Paczyński, B. 1998, ApJ, 507, L59

Li, W., Chornock, R., Leaman, J., et al. 2011, MNRAS, 412, 1473

MacFadyen, A. I., Ramirez-Ruiz, E., \& Zhang, W. 2005, tro-ph/0510192

Maihara, T., Iwamuro, F., Tanabe, H., et al. 2001, PASJ, 53, 25

Mannucci, F., Della Valle, M., Panagia, N., et al. 2005, A\&A, 433, 807

Mao, J., Wang, D., \& Bai, J. 2010, GCN Circ., 10392

Maoz, D., Mannucci, F., Li, W., et al. 2011, MNRAS, 412, 1508

McGaugh, S. S. 1991, ApJ, 380, 140

McQuinn, K. B. W., Skillman, E. D., Cannon, J. M., et al. 2009, ApJ, 695, 561

McQuinn, K. B. W., Skillman, E. D., Cannon, J. M., et al. 2010, ApJ, 724, 49

Meegan, C., Lichti, G., Bhat, P. N., et al. 2009, ApJ, 702, 791

Metzger, B. D., Martnez-Pinedo, G., Darbha, S., et al. 2010, MNRAS, 406, 2650

Metzger, B. D., Quataert, E., \& Thompson, T. A. 2008, MNRAS, 385, 1455

Miller, A. A., Perley, D. A., Bloom, J. S., Cenko, S. B., \& Nugent, P. E. 2010, GCN Circ., 10377

Modjaz, M. 2011, Astron. Nach., 332, 434

Modjaz, M., Kewley, L., Bloom, J. S., et al. 2011, ApJ, 731, L4

Modjaz, M., Kewley, L., Kirshner, R. P., et al. 2008, AJ, 135, 1136

Morgan, A. N., Cobb, B. E., Bloom, J. S., et al. 2010, GCN Circ., 10390

Moustakas, J., Kennicutt, R. C., Jr., Tremonti, C. A., et al. 2010, ApJS, 190, 233

Narayan, R., Paczyński, B., \& Piran, T. 1992, ApJ, 395, L83

Nicuesa, A., Kruehler, T., Klose, S., \& Greiner, J. 2010, GCN Circ., 10396

Noda, K., Sonoda, E., Hayasi, H., et al. 2010, GCN Circ., 10385

Nomoto, K. 1982, ApJ, 253, 798

Norris, J. P., \& Bonnell, J. T. 2006, ApJ, 643, 266

Nugent, P. E., Palomar Transient Factory, \& DeepSky Project 2009, BAAS, 41, 419

Nugent, P. E., Sullivan, M., Cenko, S. B., et al. 2011, Nature, 480, 344

Oke, J. B. 1990, AJ, 99, 1621

Oke, J. B., Cohen, J. G., Carr, M., et al. 1995, PASP, 107, 375

Oke, J. B., \& Gunn, J. E. 1983, ApJ, 266, 713

Osterbrock, D. E. 1989, Astrophysics of Gaseous Nebulae and Active Galactic Nuclei (Mill Valley, CA: University Science Books)

Paczyński, B. 1991, Acta Astron., 41, 257

Peng, C. Y., Ho, L. C., Impey, C. D., \& Rix, H.-W. 2002, AJ, 124, 266

Pérez-González, P. G., Rieke, G. H., Egami, E., et al. 2005, ApJ, 630, 82

Pettini, M., \& Pagel, B. E. J. 2004, MNRAS, 348, L59

Phillips, A. C., Miller, J., Cowley, D., \& Wallace, V. 2006, Proc. SPIE, 6269,626910

Prochaska, J. X., Bloom, J. S., Chen, H.-W., et al. 2006, ApJ, 642, 989

Reddy, N. A., Erb, D. K., Pettini, M., Steidel, C. C., \& Shapley, A. E. 2010, ApJ, 712, 1070

Roming, P. W. A., Kennedy, T. E., Mason, K. O., et al. 2005, Space Sci. Rev., 120,95

Rowlinson, A., Wiersema, K., Levan, A. J., et al. 2010, MNRAS, 408, 383

Rumyantsev, V., Shakhovkoy, D., \& Pozanenko, A. 2010, GCN Circ., 10456

Sakamoto, T., Barthelmy, S. D., Baumgartner, W. H., et al. 2010, GCN Circ., 10379

Salim, S., Dickinson, M., Michael Rich, R., et al. 2009, ApJ, 700, 161

Savaglio, S., Glazebrook, K., \& Le Borgne, D. 2009, ApJ, 691, 182

Scannapieco, E., \& Bildsten, L. 2005, ApJ, 629, L85

Schlegel, D. J., Finkbeiner, D. P., \& Davis, M. 1998, ApJ, 500, 525

Sérsic, J. L. 1963, Boletin de la Asociacion Argentina de Astronomia La Plata Argentina, 6, 41

Skrutskie, M. F., Cutri, R. M., Stiening, R., et al. 2006, AJ, 131, 1163

Stanek, K. Z., Gnedin, O. Y., Beacom, J. F., et al. 2006, Acta Astron., 56, 333

Sullivan, M., Le Borgne, D., Pritchet, C. J., et al. 2006, ApJ, 648, 868

Symeonidis, M., Willner, S. P., Rigopoulou, D., et al. 2008, MNRAS, 385, 1015

Thöne, C. C., Fynbo, J. P. U., Östlin, G., et al. 2008, ApJ, 676, 1151

Virgili, F. J., Zhang, B., O’Brien, P., \& Troja, E. 2011, ApJ, 727, 109

Wheeler, J. C. 1981, Rep. Prog. Phys., 44, 85

Woosley, S. E., \& Bloom, J. S. 2006, ARA\&A, 44, 507

Wright, E. L., Eisenhardt, P. R. M., Mainzer, A. K., et al. 2010, AJ, 140, 1868

Yasuda, N., Fukugita, M., Narayanan, V. K., et al. 2001, AJ, 122, 1104

Yoon, S., \& Langer, N. 2005, A\&A, 443, 643

Yurkov, V., Sergienko, S., Gorbovskoy, E., et al. 2010, GCN Circ., 10391

Zheng, X. Z., Hammer, F., Flores, H., Assémat, F., \& Pelat, D. 2004, A\&A, 421, 847 